\title{
Generalized Green functions associated to complex reflection groups
}

\author{
Toshiaki Shoji
}

To Michel Broué

\begin{abstract}
In this paper, we consider the set of $r$-symbols in a full generality. We construct Hall-Littlewood functions and Kostka functions associated to those $r$-symbols. We also discuss a multi-parameter version of those functions. We show that there exists a general algorithm of computing the multi-parameter Kostka functions. As an application, we show that the generalized Green functions of symplectic groups can be described combinatorially in terms of our (one-parameter) Kostka functions.
\end{abstract}

\section{INTRODUCTION}

Lusztig introduced in [L1] the notion of symbols in order to parametrize unipotent representations of finite classical groups. By using some modification of such symbols, he described in [L2] the generalized Springer correspondence for classical groups. The notion of $r$-symbols given in [Mal], [S2, 3] is a natural generalization of those symbols, where the original symbols correspond to 2-symbols. In [S2, 3], Hall-Littlewood functions and Kostka functions associated to $r$-symbols (namely, associated to complex reflection groups) were introduced. Actually, those $r$-symbols correspond to a subset of Lusztig's symbols, which is related to the Springer correspondence, and in [S2, 3], Green functions associated to complex reflection groups were discussed. In this paper, we extend the notion of $r$-symbols so that it covers the full set of symbols in the case where $r=2$. We define Hall-Littlewood functions and Kostka functions associated to those $r$-symbols, which makes it possible to define generalized Green functions associated to complex reflection groups. In [S2, 3], an algorithm of computing Kostka functions was given.

In [S4], the notion of limit symbols was introduced. It is a special case of $r$-symbols, and in fact, in that case the description of Kostka functions becomes drastically simple. It is known that those Kostka functions (in the case where $r=2$ ) are closely related to the Springer correspondence of exotic symmetric spaces $([\mathrm{AH}],[\mathrm{K}],[\mathrm{SS}])$. In $[\mathrm{S} 6]$, a multi-parameter version of Kostka functions (associated to limit symbols) was introduced. However, the algorithm of computing those Kostka functions were not given there (a straightforward generalization does not work). In this paper, we also consider the multiparameter version of Kostka functions associated to $r$-symbols of general type. We show that there exists a general algorithm of computing those Kostka functions. Although the expression is complicated in the multi-parameter case, it gives a simple algorithm in the one-parameter case. By making use of it, we show that generalized Green functions of symplectic groups can be described combinatorially by our Kostka functions. In the case of special orthogonal groups, a combinatorial description of generalized Green functions is 
also possible by using a generalization of Kostka functions defined in [S3]. We will discuss those subjects elsewhere.

\section{1. $r$-SYMBOLS}

1.1. The notion of $r$-symbols was introduced in [Mal], and was discussed in [S2, 3]. However, in those papers, some special cases of $r$-symbols were treated. In this paper, we consider the $r$-symbols in a full generality.

For an integer $n \geq 0, r \geq 1$, we denote by $\mathscr{P}_{n, r}$ the set of $r$-partitions $\boldsymbol{\lambda}=\left(\lambda^{(1)}, \ldots, \lambda^{(r)}\right)$ such that $\sum_{k=1}^{r}\left|\lambda^{(k)}\right|=n$, where $|\lambda|$ denotes the size of a partition $\lambda$. If $r=1$, we simply denote it by $\mathscr{P}_{n}$. For a partition $\lambda$, we denote by $l(\lambda)$ the number of non-zero parts of $\lambda$.

We fix a sequence $\mathbf{s}=\left(s_{1}, \ldots, s_{r}\right)$ of non-negative integers, and an integer $e$ such that $s_{k} \leq e$ for any $k$. For $\mathbf{m}=\left(m_{1}, \ldots, m_{r}\right) \in \mathbf{Z}_{\geq 0}^{r}$, we denote by $\mathscr{M}(\mathbf{m})$ the set of matrices $A=\left(a_{j}^{(k)}\right)_{1 \leq k \leq r, 1 \leq j \leq m_{k}}$ such that $a_{j}^{(k)} \in \mathbf{Z}_{\geq 0}$. We define $\widetilde{Z}^{e, \mathbf{s}}(\mathbf{m})$ as the set of $\Lambda=\left(A^{(1)}, \ldots, A^{(r)}\right) \in \mathscr{M}(\mathbf{m})$, where $A^{(k)}=\left(a_{1}^{(k)}, \ldots, a_{m_{k}}^{(k)}\right)$ is a sequence of non-negative integers satisfying the following properties;

$$
\begin{array}{ll}
a_{i}^{(k)}-a_{i+1}^{(k)} \geq e & \left(1 \leq k \leq r, 1 \leq i \leq m_{k}-1\right), \\
a_{m_{k}}^{(k)} \geq s_{k} & (1 \leq k \leq r) .
\end{array}
$$

Put $\mathbf{m}^{\prime}=\left(m_{1}+1, \ldots, m_{r}+1\right)$. We define a shift operation $\sigma: \widetilde{Z}^{e, \mathbf{s}}(\mathbf{m}) \rightarrow \widetilde{Z}^{e, \mathbf{s}}\left(\mathbf{m}^{\prime}\right)$ as follows; for $\Lambda=\left(A^{(1)}, \ldots, A^{(r)}\right) \in \widetilde{Z}^{e, \mathbf{s}}(\mathbf{m})$, define $B^{(k)}=\left(b_{1}^{(k)}, \ldots, b_{m_{k}+1}^{(k)}\right)$ by

$$
b_{i}^{(k)}= \begin{cases}a_{i}^{(k)}+e & \text { if } i \leq m_{k}, \\ s_{k} & \text { if } i=m_{k}+1 .\end{cases}
$$

If we put $\Lambda^{\prime}=\left(B^{(1)}, \ldots, B^{(r)}\right)$, then $\Lambda^{\prime} \in \widetilde{Z}^{e, \mathbf{s}}\left(\mathbf{m}^{\prime}\right)$. We define a map $\sigma: \widetilde{Z}^{e, \mathbf{s}}(\mathbf{m}) \rightarrow$ $\widetilde{Z}^{e, \mathbf{s}}\left(\mathbf{m}^{\prime}\right)$ by $\Lambda \mapsto \Lambda^{\prime}$. If we fix $\mathbf{m}$ and by applying the shift operation repeatedly, we obtain an infinite set $\bigcup_{\mathbf{m}^{\prime}} \widetilde{Z}^{e, \mathbf{s}}\left(\mathbf{m}^{\prime}\right)$ on which $\sigma$ acts. We denote by $Z_{\mathbf{d}}^{e, \mathbf{s}}$ the set of equivalence classes under $\sigma$ in this set. Here $\mathbf{d}$ denotes the equivalence class of $\mathbf{m} \in \mathbf{Z}_{\geq 0}^{r}$ under the operation $\left(a_{1}, \ldots, a_{r}\right) \mapsto\left(a_{1}+1, \ldots, a_{r}+1\right)$. A representative of $\mathbf{d}$ is given as follows; let $m$ be the smallest integer among $m_{1}, \ldots, m_{r}$, and put $d_{k}=m_{k}-m$ for each $k$. Then the sequence $\left(d_{1}, \ldots, d_{r}\right)$ determines the class $\mathbf{d}$. We denote it as $\mathbf{d}=\left(d_{1}, \ldots, d_{r}\right)$. The elements in $Z_{\mathbf{d}}^{e, \mathbf{s}}$ are called $r$-symbols of defect $\mathbf{d}$.

For $\mathbf{m}=\left(m_{1}, \ldots, m_{r}\right) \in \mathbf{Z}_{\geq 0}^{r}$, put $\Lambda^{0}(\mathbf{m})=\left(\Lambda^{(1)}, \ldots, \Lambda^{(r)}\right)$, where

$$
\Lambda^{(k)}: s_{k}+\left(m_{k}-1\right) e \geq s_{k}+\left(m_{k}-2\right) e \geq \cdots \geq s_{k}+e \geq s_{k} .
$$

1.2. We consider $\mathbf{m}^{\bullet}=\left(m_{1}, \ldots, m_{r}\right)$, where

$$
m_{1}=\cdots=m_{\alpha}=m+1, m_{\alpha+1}=\cdots=m_{r}=m
$$

for some $0 \leq \alpha<r$ and $m \geq 0$, so that the defect of $\mathbf{m}^{\bullet}$ is given by $\mathbf{d}^{\bullet}=(1, \ldots, 1,0, \ldots, 0)$ (1: $\alpha$-times). Let $\mathbf{m}^{\prime}=\left(m_{1}^{\prime}, \ldots, m_{r}^{\prime}\right)$ be such that $d_{k}^{\prime}=m_{k}^{\prime}-m_{k} \geq 0$ for any $k$ and 
$\min _{k}\left\{d_{k}^{\prime}\right\}=0$. Take $\Lambda=\left(a_{j}^{(k)}\right) \in \widetilde{Z}^{e, \mathbf{s}}\left(\mathbf{m}^{\bullet}\right)$. Put, for $k=1, \ldots, r$,

$$
B^{(k)}: a_{1}^{(k)}+d_{k}^{\prime} e \geq a_{2}^{(k)}+d_{k}^{\prime} e \geq \cdots \geq a_{m_{k}}^{(k)}+d_{k}^{\prime} e \geq s_{k}+\left(d_{k}^{\prime}-1\right) e \geq \cdots \geq s_{k}+e \geq s_{k},
$$

and $\Lambda^{\prime}=\left(B^{(1)}, \ldots, B^{(r)}\right)$. Then $\Lambda^{\prime} \in \widetilde{Z}^{e, \mathbf{s}}\left(\mathbf{m}^{\prime}\right)$. The $\operatorname{map} \Lambda \mapsto \Lambda^{\prime} ; \widetilde{Z}^{e, \mathbf{s}}\left(\mathbf{m}^{\bullet}\right) \rightarrow \widetilde{Z}^{e, \mathbf{s}}\left(\mathbf{m}^{\prime}\right)$ is compatible with the shift operation, and we obtain a map $\varphi: Z_{\mathbf{d}^{\bullet}}^{e, \mathbf{s}} \rightarrow Z_{\mathbf{d}^{\prime}}^{e, \mathbf{s}}$, where $\mathbf{d}^{\prime}$ is the defect of the class containing $\mathbf{m}^{\prime}$. Conversely, for a given $\mathbf{m}^{\prime}=\left(m_{1}^{\prime}, \ldots, m_{r}^{\prime}\right)$, we choose $\mathbf{m}^{\bullet}=\left(m_{1}, \ldots, m_{r}\right)$ such that $d_{k}^{\prime \prime}=m_{k}-m_{k}^{\prime} \geq 0$ for any $k$, with $\min _{k}\left\{d_{k}^{\prime \prime}\right\}=0$. Take $\Lambda^{\prime} \in \widetilde{Z}^{e, \mathbf{s}}\left(\mathbf{m}^{\prime}\right)=\left(b_{j}^{(k)}\right) \in \widetilde{Z}^{e, \mathbf{s}}\left(\mathbf{m}^{\prime}\right)$. Put, for $k=1, \ldots, r$,

$$
A^{(k)}: b_{1}^{(k)}+d_{k}^{\prime \prime} e \geq b_{2}^{(k)}+d_{k}^{\prime \prime} e \geq \cdots \geq b_{m_{k}^{\prime}}^{(k)}+d_{k}^{\prime \prime} e \geq s_{k}+\left(d_{k}^{\prime \prime}-1\right) e \geq \cdots \geq s_{k}+e \geq s_{k},
$$

and put $\Lambda=\left(A^{(1)}, \ldots, A^{(r)}\right)$. Then $\Lambda \in \widetilde{Z}^{e, \mathbf{s}}\left(\mathbf{m}^{\bullet}\right)$, and the map $\Lambda^{\prime} \mapsto \Lambda$ induces a map $\psi: Z_{\mathbf{d}^{\prime}}^{e, \mathbf{s}} \rightarrow Z_{\mathbf{d}^{\bullet}}^{e, \mathbf{s}}$. Since $\varphi$ and $\psi$ are inverse each other, we have a bijection $\varphi: Z_{\mathbf{d}^{\bullet}}^{e, \mathbf{s}} \stackrel{\sim}{\rightarrow} Z_{\mathbf{d}^{\prime}}^{e, \mathbf{s}}$.

For each integer $n \geq 0$, we choose $m$ such that $m \geq n$, and define $\widetilde{Z}_{n}^{e, \mathbf{s}}\left(\mathbf{m}^{\bullet}\right)$ as the subset of $\widetilde{Z}^{e, \mathbf{s}}\left(\mathbf{m}^{\bullet}\right)$ consisting of $\Lambda$ such that $\Lambda=\Lambda^{0}\left(\mathbf{m}^{\bullet}\right)+\boldsymbol{\lambda}$, where $\boldsymbol{\lambda} \in \mathscr{P}_{n, r}$ with $\boldsymbol{\lambda} \in \mathscr{M}\left(\mathbf{m}^{\bullet}\right) . \widetilde{Z}_{n}^{e, \mathbf{s}}\left(\mathbf{m}^{\bullet}\right)$ is compatible with the shift operation, and we can define a subset $Z_{n, \mathbf{d}}^{e, \mathbf{s}}$ of $Z_{\mathbf{d} \bullet}^{e, \mathbf{s}}$. Let $\mathbf{m}^{\prime}=\left(m_{1}^{\prime}, \ldots, m_{r}^{\prime}\right) \in \mathbf{Z}_{\geq 0}^{r}$ be such that $\sum_{i} m_{i}^{\prime} \equiv \alpha(\bmod r)$. We assume that $\mathbf{m}^{\prime}$ belongs to the defect $\mathbf{d}^{\prime}$. Then, by replacing $\mathbf{m}^{\prime}$ by its shift, we may assume that $d_{k}^{\prime}=m_{k}^{\prime}-m_{k} \geq 0$ with $\min _{k}\left\{d_{k}^{\prime}\right\}=0$. Put

$$
f(\mathbf{d})=\sum_{k=1}^{\alpha} d_{k}^{\prime}\left(d_{k}^{\prime}-1\right) / 2+\sum_{k=\alpha+1}^{r} d_{k}^{\prime}\left(d_{k}^{\prime}+1\right) / 2 .
$$

Using the bijection $\varphi: Z_{\mathbf{d} \bullet}^{e, \mathbf{s}} \stackrel{\sim}{\rightarrow} Z_{\mathbf{d}}^{e, \mathbf{s}}$, we define a subset $Z_{n, \mathbf{d}}^{e, \mathbf{s}}$ of $Z_{\mathbf{d}}^{e, \mathbf{s}}$ as $Z_{n, \mathbf{d}}^{e, \mathbf{s}}=\varphi\left(Z_{n^{\prime}, \mathbf{d} \bullet}^{e, \mathbf{s}}\right)$, where $n=n^{\prime}+f(\mathbf{d})$. The elements in $Z_{n, \mathbf{d}}^{e, \mathbf{s}}$ are called symbols of rank $n$ and defect d. It follows from the construction, the map $\boldsymbol{\lambda} \mapsto \varphi\left(\Lambda^{0}\left(\mathbf{m}^{\bullet}\right)+\boldsymbol{\lambda}\right)$ gives a bijection $\mathscr{P}_{n^{\prime}, r} \simeq Z_{n^{\prime}, \mathbf{d} \cdot}^{e, \mathbf{s}} \simeq Z_{n, \mathbf{d}}^{e, \mathbf{s}}$. Thus we have

(1.2.3) There is a bijection $\mathscr{P}_{n^{\prime}, r} \underset{\rightarrow}{\rightarrow} Z_{n, \mathbf{d}}^{e, \mathbf{s}}$, where $n=n^{\prime}+f(\mathbf{d})$. We denote this bijection by $\boldsymbol{\lambda} \mapsto \Lambda(\boldsymbol{\lambda})$.

For $\boldsymbol{\lambda}=\left(\lambda^{(1)}, \ldots, \lambda^{(r)}\right) \in \mathscr{P}_{n^{\prime}, r}$ as above, we fix an injective map $\theta: \mathscr{P}_{n^{\prime}, r} \rightarrow \mathscr{P}_{n, r}$. For example, we write $\lambda^{(k)}=\left(\lambda_{1}^{(k)}, \ldots, \lambda_{m_{k}^{\prime}}^{(k)}\right)$ for each $k$, and define a partition $\mu^{(k)}$ by

$$
\mu^{(k)}: \lambda_{1}^{(k)}+\left(d_{k}^{\prime}+\delta-1\right) \geq \lambda_{2}^{(k)}+\left(d_{k}^{\prime}+\delta-2\right) \geq \cdots \geq \lambda_{m_{k}^{\prime}}^{(k)}
$$

where $\delta=0$ for $1 \leq k \leq a$ and $\delta=1$ for $a+1 \leq k \leq r$. Then $\boldsymbol{\mu}=\left(\mu^{(1)}, \ldots, \mu^{(r)}\right) \in \mathscr{P}_{n, r}$. We define a map $\theta: \mathscr{P}_{n^{\prime}, r} \rightarrow \mathscr{P}_{n, r}$ by $\boldsymbol{\lambda} \mapsto \boldsymbol{\mu}$ with $\boldsymbol{\mu} \in \mathscr{M}\left(\mathbf{m}^{\prime}\right)$.

Note that for a fixed $n$, the defect $\mathbf{d}$ such that $Z_{n, \mathbf{d}}^{e, \mathbf{s}} \neq \emptyset$ is only finite by (1.2.3). Let $D$ be a subset of the set of all defects $\mathbf{d}$ such that $Z_{n, \mathbf{d}}^{e, \mathbf{s}} \neq \emptyset$. Put

$$
Z_{n, D}^{e, \mathbf{s}}=\coprod_{\mathbf{d} \in D} Z_{n, \mathbf{d}}^{e, \mathbf{s}}
$$


Hereafter, by fixing $e, \mathbf{s}$ (and $a$ in (1.2.1)), we simply write $Z_{n, D}^{e, \mathbf{s}}, Z_{n, \mathbf{d}}^{e, \mathbf{s}}$, etc., as $Z_{n, D}, Z_{n, \mathbf{d}}$, etc. if there is no fear of confusion. For a symbol $\Lambda \in Z_{n, D}$, we denote by $d(\Lambda) \in D$ the defect of $\Lambda$.

1.3. Two elements $\Lambda, \Lambda^{\prime} \in Z_{n, D}$ are called similar if for suitable representatives $\Lambda \in \widetilde{Z}_{n}(\mathbf{m}), \Lambda^{\prime} \in \widetilde{Z}_{n}\left(\mathbf{m}^{\prime}\right)$, all the components contained in $\Lambda$ and $\Lambda^{\prime}$ coincide each other with multiplicities. This gives an equivalence relation on $Z_{n, D}$, and an equivalence class in $Z_{n, D}$ is called a similarity class.

For $\mathbf{m}=\left(m_{1}, \ldots, m_{r}\right)$ such that $\sum_{i} m_{i} \equiv \alpha(\bmod r)$, we define an $a$-function $a$ : $\widetilde{Z}(\mathbf{m}) \rightarrow \mathbf{Z}$ by

$$
a(\Lambda)=\sum_{a, a^{\prime} \in \Lambda} \min \left(a, a^{\prime}\right)-\sum_{b, b^{\prime} \in \Lambda^{0}\left(\mathbf{m}^{\bullet}\right)} \min \left(b, b^{\prime}\right)
$$

where $\mathbf{m}^{\bullet}=(m+1, \ldots, m)$ is the unique element such that $\sum_{i} m_{i}=\alpha+r m$. (Note that this $\mathbf{m}^{\bullet}$ is different from $\mathbf{m}^{\bullet}$ used to define the rank of $\Lambda$ in 1.2.) If we write $\Lambda=\left(a_{j}^{(k)}\right) \in \mathscr{M}(\mathbf{m})$, we have $s_{k} \leq a_{j}^{\left(k^{\prime}\right)}+e$ for any $k, k^{\prime}, j$ by our assumption that $s_{k} \leq e$. This implies that $a(\Lambda)$ is invariant under the shift operation, and one can define $a(\Lambda)$ for $\Lambda \in Z_{n, D}$. It is clear that $a$-function is constant on each similarity class in $Z_{n, D}$.

\section{Symmetric Functions associated to $r$-PARTitions}

2.1. We fix $\mathbf{m}=\left(m_{1}, \ldots, m_{r}\right)$, and consider finitely many variables $x_{\mathbf{m}}=\left\{x_{j}^{(k)} \mid\right.$ $\left.1 \leq k \leq r, 1 \leq j \leq m_{k}\right\}$. We denote by $\Xi_{\mathbf{m}}$ the ring of symmetric polynomials

$$
\Xi_{\mathbf{m}}=\bigotimes_{k=1}^{r} \mathbf{Z}\left[x_{1}^{(k)}, \ldots, x_{m_{k}}^{(k)}\right]^{S_{m_{k}}}
$$

Let $\Xi_{\mathbf{m}}=\bigoplus_{n \geq 0} \Xi_{\mathbf{m}}^{n}$ be the decomposition of $\Xi_{\mathbf{m}}$ into homogeneous parts. Put $\mathbf{m}^{\prime}=$ $\left(m_{1}^{\prime}, \ldots, m_{r}^{\prime}\right)$ with $m_{k}^{\prime}=m_{k}+1$, and define a linear map $\rho_{\mathbf{m}, \mathbf{m}^{\prime}}^{n}: \Xi_{\mathbf{m}^{\prime}}^{n} \rightarrow \Xi_{\mathbf{m}}^{n}$ by $x_{m_{k}+1}^{(k)}=0$, and by $x_{j}^{(k)} \mapsto x_{j}^{(k)}$ for $1 \leq j \leq m_{k}$. The operation $\rho_{\mathbf{m}, \mathbf{m}^{\prime}}^{n}$ is called a shift operation on the symmetric polynomials. As the inverse limit of the $\mathbf{Z}$-module $\Xi_{\mathbf{m}}$ with respect to the shift operation $\rho_{\mathbf{m}, \mathbf{m}^{\prime}}^{n}$, we obtain

$$
\widehat{\Xi}^{n, \mathbf{d}}=\lim _{\mathbf{m}} \Xi_{\mathbf{m}}^{n}
$$

where $\mathbf{d}$ is the defect determined from $\mathbf{m}$ by the shift operation (see 1.1). The elements in $\widehat{\Xi}^{n, \mathbf{d}}$ are called symmetric functions of degree $n$ with defect $\mathbf{d}$.

For a partition $\lambda$ of $n$ with $l(\lambda) \leq m$, we can define a Schur polynomial $s_{\lambda}\left(y_{1}, \ldots, y_{m}\right)$ and a monomial symmetric polynomial $m_{\lambda}\left(y_{1}, \ldots, y_{m}\right)$. We assume that $\mathbf{m}=\left(m_{1}, \ldots, m_{r}\right)$ with $m_{k} \geq n$ for any $k$. For $\boldsymbol{\lambda}=\left(\lambda^{(1)}, \ldots, \lambda^{(r)}\right) \in \mathscr{P}_{n, r}$, we define $s_{\boldsymbol{\lambda}}\left(x_{\mathbf{m}}\right), m_{\boldsymbol{\lambda}}\left(x_{\mathbf{m}}\right) \in \Xi_{\mathbf{m}}^{n}$ by

$$
s_{\boldsymbol{\lambda}}\left(x_{\mathbf{m}}\right)=\prod_{k=1}^{r} s_{\lambda^{(k)}}\left(x_{1}^{(k)}, \ldots, x_{m_{k}}^{(k)}\right), \quad m_{\boldsymbol{\lambda}}\left(x_{\mathbf{m}}\right)=\prod_{k=1}^{r} m_{\lambda^{(k)}}\left(x_{1}^{(k)}, \ldots, x_{m_{k}}^{(k)}\right) .
$$

Then $s_{\boldsymbol{\lambda}}\left(x_{\mathbf{m}}\right), m_{\boldsymbol{\lambda}}\left(x_{\mathbf{m}}\right) \in \Xi_{\mathbf{m}}^{n}$ are compatible with the shift operation, and one can define the symmetric functions $s_{\boldsymbol{\lambda}}(x), m_{\boldsymbol{\lambda}}(x) \in \widehat{\Xi}^{n, \mathbf{d}}$. As in the classical case, the sets $\left\{s_{\boldsymbol{\lambda}} \mid \boldsymbol{\lambda} \in\right.$ 
$\left.\mathscr{P}_{n, r}\right\},\left\{m_{\boldsymbol{\lambda}} \mid \boldsymbol{\lambda} \in \mathscr{P}_{n, r}\right\}$ give the $\mathbf{Z}$-basis of $\widehat{\Xi}^{n, \mathbf{d}}$. In particular, $\widehat{\Xi}^{n, \mathbf{d}} \simeq \widehat{\Xi}^{n, \mathbf{0}}$ as $\mathbf{Z}$-modules, where $\widehat{\Xi}^{n, \mathbf{0}}$ is the symmetric functions with defect zero.

2.2. Next we define power sum symmetric functions $p_{\boldsymbol{\lambda}}(x)$. Note that the notation here is modified from the notation in [S2]. We fix a primitive $r$-th root $\zeta$ of unity in $\mathbf{C}$. Consider the variables $x_{\mathbf{m}}$ for $\mathbf{m}=\left(m_{1}, \ldots, m_{r}\right)$. We define, for each integer $s \geq 1$ and $1 \leq k \leq r$, a symmetric polynomial $p_{s}^{(k)}\left(x_{\mathbf{m}}\right)$ by

$$
p_{s}^{(k)}\left(x_{\mathbf{m}}\right)=\sum_{j=1}^{r} \zeta^{(k-1)(j-1)} p_{s}\left(x_{1}^{(j)}, \ldots, x_{m_{j}}^{(j)}\right),
$$

where $p_{s}\left(y_{1}, \ldots, y_{m}\right)=\sum_{i} y_{i}^{s}$ is the classical power sum symmetric polynomial. Put $p_{s}\left(x_{\mathbf{m}}\right)=1$ for $s=0$. For each $\boldsymbol{\lambda} \in \mathscr{P}_{n, r}$, we define a polynomial $p_{\boldsymbol{\lambda}}\left(x_{\mathbf{m}}\right)$ by

$$
p_{\boldsymbol{\lambda}}\left(x_{\mathbf{m}}\right)=\prod_{k=1}^{r} \prod_{j=1}^{m_{k}} p_{\lambda_{j}^{(k)}}^{(k)}\left(x_{\mathbf{m}}\right)
$$

Then $p_{\boldsymbol{\lambda}} \in \Xi_{\mathbf{m}}^{n} \otimes_{\mathbf{Z}} \mathbf{C}$, compatible with the shift operation, and one can define a symmetric function $p_{\boldsymbol{\lambda}}(x) .\left\{p_{\boldsymbol{\lambda}} \mid \boldsymbol{\lambda} \in \mathscr{P}_{n, r}\right\}$ gives a $\mathbf{C}$-basis of $\widehat{\Xi}^{n, \mathbf{d}} \otimes_{\mathbf{Z}} \mathbf{C}$.

2.3. Following [S6], we introduce $r$ parameters $t_{1}, \ldots, t_{r}$, and consider the symmetric functions with parameters $\mathbf{t}=\left(t_{1}, \ldots, t_{r}\right)$. Put $\mathbf{Z}[\mathbf{t}]=\mathbf{Z}\left[t_{1}, \ldots, t_{r}\right]$, the polynomial ring with variables $t_{1}, \ldots, t_{r}$, and $\mathbf{Q}(\mathbf{t})=\mathbf{Q}\left(t_{1}, \ldots, t_{r}\right)$ the field of rational functions with variables $t_{1}, \ldots, t_{r}$. We denote $\Xi_{\mathbf{m}}^{n} \otimes_{\mathbf{Z}} \mathbf{Z}[\mathbf{t}], \widehat{\Xi}^{n, \mathbf{d}} \otimes_{\mathbf{Z}} \mathbf{Z}[\mathbf{t}]$, etc., by $\Xi_{\mathbf{m}}^{n}[\mathbf{t}], \widehat{\Xi}^{n, \mathbf{d}}[\mathbf{t}]$, etc.. Also put $\widehat{\Xi}^{n, \mathbf{d}} \otimes_{\mathbf{Z}} \mathbf{Q}(\mathbf{t})=\widehat{\Xi}_{\mathbf{Q}}^{n, \mathbf{d}}(\mathbf{t})$ and $\widehat{\Xi}^{n, \mathbf{d}} \otimes_{\mathbf{Z}} \mathbf{C}(\mathbf{t})=\widehat{\Xi}_{\mathbf{C}}^{n, \mathbf{d}}(\mathbf{t})$. We consider the one-parameter case also, which we denote by $\Xi_{\mathbf{m}}^{n}[t]$, etc. for the parameter $t$. We use the notation $\mathbf{t}_{0}=(t, \ldots, t)$, so that the specialization $\mathbf{t} \mapsto \mathbf{t}_{0}$ gives $\Xi_{\mathbf{m}}^{n}\left[\mathbf{t}_{0}\right]=\Xi_{\mathbf{m}}^{n}[t]$.

In the rest of this section, we assume that $\mathbf{d}=\mathbf{0}$, namely $\mathbf{m}=(m, \ldots, m)$ for some $m>0$.

Following [S2], we introduce polynomials (with one-parameter $t) q_{s, \pm}^{(k)}\left(x_{\mathbf{m}} ; t\right) \in \Xi_{\mathbf{m}}^{s}[t]$. (But note that the definition here is modified from (2.2.1) in [S2], based on Remark 5.7 in [S3]. Also note that since we assume that $\mathbf{d}=\mathbf{0}$, the discussion is simplified from there). For any integer $s \geq 1$, we define (depending on the signature "+" or "-"),

$$
q_{s, \pm}^{(k)}\left(x_{\mathbf{m}} ; t\right)=\sum_{i=1}^{m}\left(x_{i}^{(k)}\right)^{s-1} \frac{\prod_{j \geq 1}\left(x_{i}^{(k)}-t x_{j}^{(k \mp 1)}\right)}{\prod_{j \neq i}\left(x_{i}^{(k)}-x_{j}^{(k)}\right)},
$$

and put $q_{s, \pm}^{(k)}\left(x_{\mathbf{m}} ; t\right)=1$ if $s=0$. Note in the formula (2.3.1), we regard $k \mp 1$ as an element in $\mathbf{Z} / r \mathbf{Z}$. Let $u$ be another variable. By using Lagrange's interpolation, we have a formula (see [S2, Lemma 2.3])

$$
\prod_{i=1}^{m} \frac{1-t u x_{i}^{(k \mp 1)}}{1-u x_{i}^{(k)}}=1+\sum_{i=1}^{m} \frac{u x_{i}^{(k)}-t u x_{i}^{(k \mp 1)}}{1-u x_{i}^{(k)}} \prod_{j \neq i} \frac{x_{i}^{(k)}-t x_{j}^{(k \mp 1)}}{x_{i}^{(k)}-x_{j}^{(k)}} .
$$


It follows from this that

$$
\sum_{s=0}^{\infty} q_{s, \pm}^{(k)}\left(x_{\mathbf{m}} ; t\right) u^{s}=\prod_{i=1}^{m} \frac{1-t u x_{i}^{(k \mp 1)}}{1-u x_{i}^{(k)}} .
$$

In particular, we see that $q_{s, \pm}^{(k)}\left(x_{\mathbf{m}} ; t\right) \in \Xi_{\mathbf{m}}^{s}[t]$. One can check that $q_{s, \pm}^{(k)}\left(x_{\mathbf{m}} ; t\right)$ is compatible with the shift operation.

Following [S6], we define, for $\boldsymbol{\lambda} \in \mathscr{P}_{n, r}$, a polynomial $q_{\boldsymbol{\lambda}}^{ \pm}\left(x_{\mathbf{m}} ; \mathbf{t}\right)$ by

$$
q_{\boldsymbol{\lambda}}^{ \pm}\left(x_{\mathbf{m}} ; \mathbf{t}\right)=\prod_{k \in \mathbf{Z} / r \mathbf{Z}} \prod_{i=1}^{m} q_{\lambda_{i}^{(k)}, \pm}^{(k)}\left(x_{\mathbf{m}} ; t_{k-c}\right),
$$

where $c=1$ for the "+"-case, and $c=0$ for the "-" -case. We see that $q_{\boldsymbol{\lambda}}^{ \pm} \in \Xi_{\mathbf{m}}^{n}[\mathbf{t}]$, and $q_{\boldsymbol{\lambda}}^{ \pm}$is compatible with the shift operation. Hence we obtain symmetric functions $q_{\boldsymbol{\lambda}}^{ \pm}(x ; \mathbf{t}) \in \Xi^{n}[\mathbf{t}]$. Note that $q_{\boldsymbol{\lambda}}^{ \pm}\left(x ; \mathbf{t}_{0}\right)$ coincides with $q_{\boldsymbol{\lambda}}^{ \pm}(x ; t)$ introduced in [S2, 2.2].

2.4. We prepare two types of infinitely many variables $x=\left(x^{(1)}, \ldots, x^{(r)}\right)$ and $y=\left(y^{(1)}, \ldots, y^{(r)}\right)$, and consider the product

$$
\Omega(x, y ; \mathbf{t})=\prod_{k \in \mathbf{Z} / r \mathbf{Z}} \prod_{i, j} \frac{1-t_{k} x_{i}^{(k)} y_{j}^{(k+1)}}{1-x_{i}^{(k)} y_{j}^{(k)}} .
$$

(Note that this formula was introduced in [S6]. A one-parameter version appears in [S2, Prop. 2.5], but the present version is modified from there, following Remark 5.7 in [S3]). The following formula appears in [S6, Prop. 2.8], which is a multi-parameter version of [S2, Prop. 2.5.], and is proved similarly.

Proposition 2.5. Under the notation above, the following formulas hold.

$$
\Omega(x, y ; \mathbf{t})=\sum_{\boldsymbol{\lambda}} q_{\boldsymbol{\lambda}}^{+}(x ; \mathbf{t}) m_{\boldsymbol{\lambda}}(y)=\sum_{\boldsymbol{\lambda}} m_{\boldsymbol{\lambda}}(x) q_{\boldsymbol{\lambda}}^{-}(y ; \mathbf{t})
$$

where $\boldsymbol{\lambda}$ runs over all the r-partitions.

2.6. In the one-parameter case, $\Omega(x, y ; t)$ is also expressed by using power sum symmetric functions as in $[\mathrm{S} 2,(2.5 .2)]$. We shall show, in the multi-parameter case, still $\Omega(x, y, \mathbf{t})$ can be expressed by power sum symmetric functions.

If we denote by $p_{m}^{[k]}(x)$ the power sum symmetric function $\sum_{i}\left(x_{i}^{(k)}\right)^{m}$ with respect to the variables $x^{(k)},(2.2 .1)$ implies that

$$
p_{m}^{(a)}(x)=\sum_{1 \leq k \leq r} \zeta^{(a-1)(k-1)} p_{m}^{[k]}(x) .
$$


We consider a matrix $\Delta=\left(\zeta^{(a-1)(k-1)}\right)_{1 \leq a, k \leq r}$ with $\operatorname{det} \Delta=\prod_{0 \leq j<i<r}\left(\zeta^{i}-\zeta^{j}\right) \neq 0$. Let $S(\mathbf{t})=\operatorname{Diag}\left(t_{1}, \ldots, t_{r}\right)$ be a diagonal matrix of degree $r$, and put

$$
\Delta(\mathbf{t})=\Delta S(\mathbf{t}) \Delta^{-1}
$$

We write $\Delta(\mathbf{t})=\left(\Delta_{a, a^{\prime}}(\mathbf{t})\right)_{1 \leq a, a^{\prime} \leq r}$, where $\Delta_{a, a^{\prime}}(\mathbf{t}) \in \mathbf{C}(\mathbf{t})$. For any integer $m \geq 0$, put $\mathbf{t}^{m}=\left(t_{1}^{m}, \ldots, t_{r}^{m}\right)$. Then we have

$$
\sum_{k} t_{k}^{m} \zeta^{(a-1)(k-1)} p_{m}^{[k]}(x)=\sum_{1 \leq a^{\prime} \leq r} \Delta_{a, a^{\prime}}\left(\mathbf{t}^{m}\right) p_{m}^{\left(a^{\prime}\right)}(x)
$$

Let $\mathscr{P}_{n, r}^{2}$ be the set of $\boldsymbol{\xi}=\left(\xi^{(i, j)}\right)_{1 \leq i, j \leq r}$, where $\xi^{(i, j)}$ are partitions such that $\sum_{i, j}\left|\xi^{(i, j)}\right|=$ $n$. For $\boldsymbol{\xi} \in \mathscr{P}_{n, r}^{2}$, we define $\boldsymbol{\xi}^{\prime}=\left(\xi^{\prime(1)}, \ldots, \xi^{\prime(r)}\right) \in \mathscr{P}_{n, r}$ by $\xi^{\prime(k)}=\bigcup_{1 \leq j \leq r} \xi^{(k, j)}$, and define $\boldsymbol{\xi}^{\prime \prime}=\left(\xi^{\prime \prime(1)}, \ldots, \xi^{\prime \prime(r)}\right) \in \mathscr{P}_{n, r}$ by $\xi^{\prime \prime(k)}=\bigcup_{1 \leq i \leq r} \xi^{(i, k)}$. (Here for partitions $\lambda$, $\mu$, we denote by $\lambda \cup \mu$ the partition of $|\lambda|+|\mu|$ obtained by rearranging the parts of $\lambda$ and $\mu$ in a decreasing order). For $\boldsymbol{\xi} \in \mathscr{P}_{n, r}^{2}$, we define $z_{\boldsymbol{\xi}}(\mathbf{t})$ by

$$
z_{\boldsymbol{\xi}}(\mathbf{t})=z_{\boldsymbol{\xi}}^{-1} \prod_{1 \leq a, a^{\prime} \leq r} z_{\xi^{\left(a, a^{\prime}\right)}}(\mathbf{t})
$$

where, for $\xi^{\left(a, a^{\prime}\right)}=\left(\xi_{1}, \xi_{2}, \ldots, \xi_{\ell}\right)$ with $\ell=l\left(\xi^{\left(a, a^{\prime}\right)}\right)$,

$$
z_{\xi^{\left(a, a^{\prime}\right)}}(\mathbf{t})= \begin{cases}\prod_{j=1}^{\ell}\left(\delta_{a, a^{\prime}}-\zeta^{a-1} \Delta_{a, a^{\prime}}\left(\mathbf{t}^{\xi_{j}}\right)\right) & \text { if } \ell>0 \\ 1 & \text { if } \ell=0 .\end{cases}
$$

Moreover $z_{\boldsymbol{\xi}}$ is defined as follows; for a partition $\lambda=\left(1^{m_{1}}, 2^{m_{2}}, \ldots\right)$, put $z_{\lambda}=\prod_{i \geq 1} i^{m_{i}} m_{i}$ !. Then $z_{\boldsymbol{\xi}}=r^{l(\boldsymbol{\xi})} \prod_{1 \leq a, a^{\prime} \leq r} z_{\xi^{\left(a, a^{\prime}\right)}}$, where $l(\boldsymbol{\xi})=\sum_{a, a^{\prime}} l\left(\xi^{\left(a, a^{\prime}\right)}\right)$.

Remark 2.7. $z_{\xi^{\left(a, a^{\prime}\right)}}\left(\mathbf{t}_{0}\right)$ is zero unless $a=a^{\prime}$, hence $z_{\boldsymbol{\xi}}\left(\mathbf{t}_{0}\right)=0$ unless $\boldsymbol{\xi}=\left(\xi^{\left(a, a^{\prime}\right)}\right)$ with $\xi^{\left(a, a^{\prime}\right)}=\emptyset$ for any pair $a \neq a^{\prime}$. Thus if $z_{\boldsymbol{\xi}}\left(\mathbf{t}_{0}\right) \neq 0$, then $\boldsymbol{\xi}$ is identified with an $r$-partition $\boldsymbol{\lambda}=\left(\lambda^{(1)}, \ldots, \lambda^{(r)}\right)$ with $\lambda^{(k)}=\xi^{(k, k)}$ for each $k$. In this case, $z_{\boldsymbol{\xi}}\left(\mathbf{t}_{0}\right)$ coincides with $z_{\boldsymbol{\lambda}}(t)^{-1}$ defined in $[\mathrm{S} 2,2.4]$, where

$$
z_{\boldsymbol{\lambda}}(t)=z_{\boldsymbol{\lambda}} \prod_{k=1}^{r} z_{\lambda^{(k)}}(t)
$$

with

$$
z_{\lambda^{(k)}}(t)=\prod_{j=1}^{l\left(\lambda^{(k)}\right)}\left(1-\zeta^{k-1} t^{\lambda_{j}^{(k)}}\right)^{-1}
$$

Proposition 2.8. Under the notation above, we have

$$
\Omega(x, y ; \mathbf{t})=\sum_{\boldsymbol{\xi}} z_{\boldsymbol{\xi}}(\mathbf{t}) p_{\boldsymbol{\xi}^{\prime}}(x) \bar{p}_{\boldsymbol{\xi}^{\prime \prime}}(y)
$$


where $\boldsymbol{\xi}$ runs over all the elements in $\mathscr{P}_{n, r}^{2}$ for any $n \geq 0$, and $\bar{p}_{\boldsymbol{\xi}^{\prime \prime}}(y)$ denotes the complex conjugate of $p_{\boldsymbol{\xi}^{\prime \prime}}(y)$.

Proof. We can write as

$$
\log \Omega(x, y ; \mathbf{t})=\sum_{k} \sum_{i, j} \sum_{m=1}^{\infty}\left(\frac{1}{m}\left(x_{i}^{(k)} y_{j}^{(k)}\right)^{m}-\frac{t_{k}^{m}}{m}\left(x_{i}^{(k)} y_{j}^{(k+1)}\right)^{m}\right) .
$$

By using the orthogonality relations of irreducible characters of $\mathbf{Z} / r \mathbf{Z}$,

$$
\frac{1}{r} \sum_{a=1}^{r}\left(\zeta^{k} \zeta^{-k^{\prime}}\right)^{a}=\delta_{k . k^{\prime}}
$$

the right hand side of (2.8.2) can be rewritten as

$$
\begin{aligned}
\sum_{m=1}^{\infty} \sum_{a=1}^{r} \sum_{i, j, k, k^{\prime}} & \left(\frac{1}{r m} \zeta^{(a-1)(k-1)} \zeta^{-(a-1)\left(k^{\prime}-1\right)}\left(x_{i}^{(k)} y_{j}^{\left(k^{\prime}\right)}\right)^{m}\right. \\
& \left.-\frac{t_{k}^{m}}{r m} \zeta^{(a-1)} \zeta^{(a-1)(k-1)} \zeta^{-(a-1)\left(k^{\prime}\right)}\left(x_{i}^{(k)} y_{j}^{\left(k^{\prime}+1\right)}\right)^{m}\right) .
\end{aligned}
$$

By using the definition (2.2.1) of power sum symmetric functions, we have

$$
\sum_{k, k^{\prime}, i, j} \frac{1}{r m} \zeta^{(a-1)(k-1)} \zeta^{-(a-1)\left(k^{\prime}-1\right)}\left(x_{i}^{(k)} y_{j}^{\left(k^{\prime}\right)}\right)^{m}=\frac{1}{r m} p_{m}^{(a)}(x) \overline{p_{m}^{(a)}(y)} .
$$

On the other hand, by using (2.6.2), we have

$$
\begin{aligned}
\sum_{k, k^{\prime}, i, j} \frac{t_{k}^{m}}{r m} \zeta^{(a-1)} \zeta^{(a-1)(k-1)} \zeta^{-(a-1)\left(k^{\prime}\right)}\left(x_{i}^{(k)} y_{j}^{\left(k^{\prime}+1\right)}\right)^{m} \\
=\sum_{1 \leq a^{\prime} \leq r} \frac{\zeta^{a-1}}{r m} \Delta_{a, a^{\prime}}\left(\mathbf{t}^{m}\right) p_{m}^{\left(a^{\prime}\right)}(x) \overline{p_{m}^{(a)}(y)}
\end{aligned}
$$

Thus (2.8.3) can be written as

$$
\sum_{1 \leq a, a^{\prime} \leq r} \sum_{m=1}^{\infty} \frac{\delta_{a, a^{\prime}}-\zeta^{a-1} \Delta_{a, a^{\prime}}\left(\mathbf{t}^{m}\right)}{r m} p_{m}^{\left(a^{\prime}\right)}(x) \overline{p_{m}^{(a)}(y)} .
$$

Hence we have

$$
\Omega(x, y ; \mathbf{t})=\prod_{a=1}^{r} \prod_{a^{\prime}=1}^{r} \prod_{m=1}^{\infty} \exp \left(\frac{\delta_{a, a^{\prime}}-\zeta^{a-1} \Delta_{a, a^{\prime}}\left(\mathbf{t}^{m}\right)}{r m} p_{m}^{\left(a^{\prime}\right)}(x) \overline{p_{m}^{(a)}(y)}\right) .
$$


This implies that

$$
\Omega(x, y ; \mathbf{t})=\sum_{\boldsymbol{\xi}} z_{\boldsymbol{\xi}}(\mathbf{t}) \prod_{a, a^{\prime}} \prod_{j} p_{\xi_{j}}^{\left(a^{\prime}\right)}(x) \overline{p_{\xi_{j}}^{(a)}(y)}
$$

where $\boldsymbol{\xi}$ runs over all elements in $\bigcup_{n \geq 0} \mathscr{P}_{n, r}^{2}$, and we write $\boldsymbol{\xi}=\left(\xi^{\left(a, a^{\prime}\right)}\right)$ with $\xi^{\left(a, a^{\prime}\right)}=$ $\left(\xi_{1}, \ldots, \xi_{k}\right)$. In view of the discussion in 2.6 , the proposition follows from (2.8.7).

2.9. For any $\boldsymbol{\lambda}, \boldsymbol{\mu} \in \mathscr{P}_{n, r}$, put

$$
z_{\boldsymbol{\lambda}, \boldsymbol{\mu}}(\mathbf{t})=\sum_{\substack{\boldsymbol{\xi} \in \mathscr{P}_{n, r}^{2} \\ \boldsymbol{\xi}^{\prime}=\boldsymbol{\lambda}, \boldsymbol{\xi}^{\prime \prime}=\boldsymbol{\mu}}} z_{\boldsymbol{\xi}}(\mathbf{t})
$$

Then (2.8.1) can be rewritten as follows.

$$
\Omega(x, y ; \mathbf{t})=\sum_{\boldsymbol{\lambda}, \boldsymbol{\mu}} z_{\boldsymbol{\lambda}, \boldsymbol{\mu}}(\mathbf{t}) p_{\boldsymbol{\lambda}}(x) \bar{p}_{\boldsymbol{\mu}}(y)
$$

where $\boldsymbol{\lambda}, \boldsymbol{\mu}$ run over all $r$-partitions.

By Remark 2.7, $z_{\boldsymbol{\lambda}, \boldsymbol{\mu}}\left(\mathbf{t}_{0}\right)=z_{\boldsymbol{\lambda}}(t)^{-1}$ if $\boldsymbol{\lambda}=\boldsymbol{\mu}$, and is equal to zero otherwise. Hence (2.9.2) implies that

$$
\Omega\left(x, y ; \mathbf{t}_{0}\right)=\Omega(x, y ; t)=\sum_{\boldsymbol{\lambda}} z_{\boldsymbol{\lambda}}(t)^{-1} p_{\boldsymbol{\lambda}}(x) \bar{p}_{\boldsymbol{\lambda}}(y)
$$

which coincides with the formula in $[\mathrm{S} 2,(2.5 .2)]$.

2.10. It follows from 2.1 that $\left\{s_{\boldsymbol{\lambda}} \mid \boldsymbol{\lambda} \in \mathscr{P}_{n, r}\right\},\left\{m_{\boldsymbol{\lambda}} \mid \boldsymbol{\lambda} \in \mathscr{P}_{n, r}\right\}$ give the $\mathbf{Z}[\mathbf{t}]$ basis of $\mathbf{Z}[\mathbf{t}]$-module $\widehat{\Xi}^{n, 0}[\mathbf{t}]$. On the other hand, it is known by [S6, Lemma 2.6] that $\left\{q_{\boldsymbol{\lambda}}^{ \pm} \mid \boldsymbol{\lambda} \in \mathscr{P}_{n, r}\right\}$ gives a $\mathbf{Q}(\mathbf{t})$-basis of $\widehat{\Xi}_{\mathbf{Q}}^{n, \mathbf{0}}(\mathbf{t})$. We define a non-degenerate bilinear form $\langle\rangle:, \widehat{\Xi}_{\mathbf{Q}}^{n, \mathbf{0}}(\mathbf{t}) \times \widehat{\Xi}_{\mathbf{Q}}^{n, \mathbf{0}}(\mathbf{t}) \rightarrow \mathbf{Q}(\mathbf{t})$ by

$$
\left\langle q_{\boldsymbol{\lambda}}^{+}(x ; \mathbf{t}), m_{\boldsymbol{\mu}}(x)\right\rangle=\delta_{\boldsymbol{\lambda}, \boldsymbol{\mu}}
$$

By using a similar argument as in [Mac, Chap.1, 4], we obtain the following result as a corollary of Proposition 2.5.

$$
\left\langle m_{\boldsymbol{\lambda}}(x), q_{\boldsymbol{\mu}}^{-}(x ; \mathbf{t})\right\rangle=\delta_{\boldsymbol{\lambda}, \boldsymbol{\mu}} .
$$

\section{Hall-Littlewood functions associated to $r$-Symbols}

3.1. Hall-Littlewood functions associated to $r$-symbols were constructed in [S2], but the discussion there was focussed on the symbols of fixed defect, namely the symbols in connection with the Springer correspondence. In order to extend it to the case of arbitrary 
defects, we will reformulate the construction of Hall-Littlewood functions in [S2], based on the discussion in [S6].

3.2. Take $\mathbf{m}=\left(m_{1}, \ldots, m_{r}\right)$, and let $\mathbf{d}=\left(d_{1}, \ldots, d_{r}\right), \mathbf{m}^{\bullet}=(m, \ldots, m)$ be as in 1.1, 1.2. For a given integer $n \geq 0$, put $n^{\prime}=n-f(\mathbf{d})$. Let $\theta: \mathscr{P}_{n^{\prime}, r} \rightarrow \mathscr{P}_{n, r}$ be the map defined in 1.2. By noticing that $\left\{s_{\boldsymbol{\lambda}} \mid \boldsymbol{\lambda} \in \mathscr{P}_{n^{\prime}, r}\right\}$ is a basis of $\widehat{\Xi}^{n^{\prime}, \mathbf{0}}[\mathbf{t}]$, we define a map $\Theta=\Theta_{\mathbf{d}}: \widehat{\Xi}^{n^{\prime}, \mathbf{0}}[\mathbf{t}] \rightarrow \widehat{\Xi}^{n, \mathbf{d}}[\mathbf{t}]$ by $s_{\boldsymbol{\lambda}}(x) \mapsto s_{\theta(\boldsymbol{\lambda})}(x)$. (Here we regard $\boldsymbol{\lambda} \in \mathscr{M}\left(\mathbf{m}^{\bullet}\right)$ as an element in $\mathscr{M}(\mathbf{m})$ by a suitable addition of 0's.) $\Theta$ gives an embedding $\widehat{\Xi}^{n^{\prime}, \mathbf{0}}[\mathbf{t}] \hookrightarrow \widehat{\Xi}^{n, \mathbf{d}}[\mathbf{t}]$. We denote by $\Xi^{n, \mathbf{d}}[\mathbf{t}]$ the image of $\Theta$. We also put $\Xi_{\mathbf{Q}}^{n, \mathbf{d}}(\mathbf{t})=\Xi^{n, \mathbf{d}}[\mathbf{t}] \otimes_{\mathbf{Z}[\mathbf{t}]} \mathbf{Q}(\mathbf{t}), \Xi_{\mathbf{C}}^{n, \mathbf{d}}(\mathbf{t})=$ $\Xi^{n, \mathbf{d}}[\mathbf{t}] \otimes_{\mathbf{Z}[\mathbf{t}]} \mathbf{C}(\mathbf{t}) . \Theta$ can be extended to the map on these spaces, which we also denote by $\Theta$. By (1.2.3), the map $\boldsymbol{\lambda} \mapsto \Lambda(\boldsymbol{\lambda})=\varphi\left(\Lambda^{0}\left(\mathbf{m}^{\bullet}\right)+\boldsymbol{\lambda}\right)$ gives a bijective correspondence $\mathscr{P}_{n^{\prime}, r} \underset{\rightarrow}{\rightarrow} Z_{n, \mathbf{d}}$. For each $\boldsymbol{\lambda} \in \mathscr{P}_{n^{\prime}, r}$, we define $m_{\Lambda(\boldsymbol{\lambda})}, q_{\Lambda(\boldsymbol{\lambda})}^{ \pm}$by $m_{\Lambda(\boldsymbol{\lambda})}=\Theta\left(m_{\boldsymbol{\lambda}}\right), q_{\Lambda(\boldsymbol{\lambda})}^{ \pm}=$ $\Theta\left(q_{\boldsymbol{\lambda}}^{ \pm}\right)$. Also put $s_{\Lambda(\boldsymbol{\lambda})}=\Theta\left(s_{\boldsymbol{\lambda}}\right)$. Then $\left\{s_{\Lambda} \mid \Lambda \in Z_{n, \mathbf{d}}\right\},\left\{m_{\Lambda} \mid \Lambda \in Z_{n, \mathbf{d}}\right\}$ give $\mathbf{Z}[\mathbf{t}]$-bases of $\Xi^{n, \mathbf{d}}[\mathbf{t}]$. Moreover, $\left\{q_{\Lambda}^{ \pm} \mid \Lambda \in Z_{n, \mathbf{d}}\right\}$ gives a $\mathbf{Q}(\mathbf{t})$-basis of $\Xi_{\mathbf{Q}}^{n, \mathbf{d}}(\mathbf{t})$. On the other hand, if we put $p_{\Lambda(\boldsymbol{\lambda})}=\Theta\left(p_{\boldsymbol{\lambda}}\right)$, then $\left\{p_{\Lambda} \mid \Lambda \in Z_{n, \mathbf{d}}\right\}$ gives a $\mathbf{C}(\mathbf{t})$-basis of $\Xi_{\mathbf{C}}^{n, \mathbf{d}}(\mathbf{t})$.

For $\Lambda=\Lambda(\boldsymbol{\lambda}), \Lambda^{\prime}=\Lambda(\boldsymbol{\mu})$ with $\boldsymbol{\lambda}, \boldsymbol{\mu} \in \mathscr{P}_{n^{\prime}, r}$, put $z_{\Lambda, \Lambda^{\prime}}(\mathbf{t})=z_{\boldsymbol{\lambda}, \boldsymbol{\mu}}(\mathbf{t})$ (see (2.9.1)). We define $\Omega^{n, \mathbf{d}}(x, y ; \mathbf{t})$ by

$$
\Omega^{n, \mathbf{d}}(x, y ; \mathbf{t})=\sum_{\Lambda, \Lambda^{\prime} \in Z_{n, \mathbf{d}}} z_{\Lambda, \Lambda^{\prime}}(\mathbf{t}) p_{\Lambda}(x) \bar{p}_{\Lambda^{\prime}}(y)
$$

Note that $\bar{p}_{\Lambda(\boldsymbol{\lambda})}=\Theta\left(\bar{p}_{\boldsymbol{\lambda}}\right)$ since $\Theta$ is defined over $\mathbf{Q}(\mathbf{t})$. Thus by Proposition 2.5 and (2.9.2), we have

Proposition 3.3. Under the notation as above,

$$
\Omega^{n, \mathbf{d}}(x, y ; \mathbf{t})=\sum_{\Lambda \in Z_{n, \mathbf{d}}} q_{\Lambda}^{+}(x ; \mathbf{t}) m_{\Lambda}(y)=\sum_{\Lambda \in Z_{n, \mathbf{d}}} m_{\Lambda}(x) q_{\Lambda}^{-}(y ; \mathbf{t})
$$

3.4. Put $\Xi_{\mathbf{Q}}^{n, D}(\mathbf{t})=\bigoplus_{\mathbf{d} \in D} \Xi_{\mathbf{Q}}^{n, \mathbf{d}}(\mathbf{t})$, where $D$ is as in 1.2. Then $\left\{s_{\Lambda} \mid \Lambda \in Z_{n, D}\right\}$, $\left\{m_{\Lambda} \mid \Lambda \in Z_{n, D}\right\}$ and $\left\{q_{\Lambda}^{ \pm} \mid \Lambda \in Z_{n, D}\right\}$ give bases of $\Xi_{\mathbf{Q}}^{n, D}(\mathbf{t})$. If we define $\Xi_{\mathbf{C}}^{n, D}(\mathbf{t})$ similarly, $\left\{p_{\Lambda} \mid \Lambda \in Z_{n, D}\right\}$ gives a basis of $\Xi_{\mathbf{C}}^{n, D}(\mathbf{t})$. We define a bilinear form $\langle$,$\rangle on \Xi_{\mathbf{Q}}^{n, \mathbf{d}}(\mathbf{t})$ by making use of the isomorphism $\Theta_{\mathbf{d}}: \Xi_{\mathbf{Q}}^{n^{\prime}, \mathbf{0}}(\mathbf{t}) \underset{\sim}{\rightarrow} \Xi_{\mathbf{Q}}^{n, \mathbf{d}}(\mathbf{t})$, then extend it to the bilinear form on $\Xi_{\mathbf{Q}}^{n, D}(\mathbf{t})$ subject to the condition that $\Xi_{\mathbf{Q}}^{n, \mathbf{d}}(\mathbf{t})$ and $\Xi_{\mathbf{Q}}^{n, \mathbf{d}^{\prime}}(\mathbf{t})$ are orthogonal if $\mathbf{d} \neq \mathbf{d}^{\prime}$. Also we extend this to the sesqui linear form on $\Xi_{\mathbf{C}}^{n, D}(\mathbf{t})$. Then by (2.10.1) and (2.10.2), we have

$$
\left\langle q_{\Lambda}^{+}(x ; \mathbf{t}), m_{\Lambda^{\prime}}(x)\right\rangle=\delta_{\Lambda, \Lambda^{\prime}}, \quad\left\langle m_{\Lambda}(x), q_{\Lambda^{\prime}}^{-}(x ; \mathbf{t})\right\rangle=\delta_{\Lambda, \Lambda^{\prime}} .
$$

The isomorphism $\Theta_{\mathbf{d}}: \widehat{\Xi}_{\mathbf{Q}}^{n^{\prime}, \mathbf{0}}(\mathbf{t}) \stackrel{\sim}{\rightarrow} \Xi_{\mathbf{Q}}^{n, \mathbf{d}}(\mathbf{t})$ makes sense for $\mathbf{t}=\mathbf{0}$, and we can define a Q-vector space $\Xi_{\mathbf{Q}}^{n, \mathbf{d}}=\Theta_{\mathbf{d}}\left(\Xi_{\mathbf{Q}}^{n^{\prime}, \mathbf{0}}\right)$. Put $\Xi_{\mathbf{Q}}^{n, D}=\bigoplus_{\mathbf{d} \in D} \Xi_{\mathbf{Q}}^{n, \mathbf{d}}$. Then $\left\{s_{\Lambda} \mid \Lambda \in Z_{n, D}\right\}$ gives a Q-basis of $\Xi_{\mathbf{Q}}^{n, D}$. By applying the discussion in [Mac, 1.4] for variables $x^{(k)}, y^{(k)}$ in the 
case where $\mathbf{t}=\mathbf{0}$, we obtain the formula

$$
\Omega(x, y ; \mathbf{0})=\prod_{1 \leq k \leq r} \prod_{i, j}\left(1-x_{i}^{(k)} y_{j}^{(k)}\right)^{-1}=\sum_{\boldsymbol{\lambda}} s_{\boldsymbol{\lambda}}(x) s_{\boldsymbol{\lambda}}(y) .
$$

Thus, by applying $\Theta_{\mathbf{d}}$ for each $\mathbf{d}$, we have

$$
\Omega^{n, \mathbf{d}}(x, y ; \mathbf{0})=\sum_{\Lambda \in Z_{n, \mathbf{d}}} s_{\Lambda}(x) s_{\Lambda}(y)
$$

We define a symmetric bilinear form $\langle,\rangle_{0}$ on $\Xi_{\mathbf{Q}}^{n, D}$ by $\left\langle s_{\Lambda}, s_{\Lambda^{\prime}}\right\rangle_{0}=\delta_{\Lambda, \Lambda^{\prime}}\left(\Lambda, \Lambda^{\prime} \in Z_{n, D}\right)$.

Let $\mathscr{A}$ be the $\mathbf{Q}[\mathbf{t}]$-subalgebra of $\mathbf{Q}(\mathbf{t})$ generated by rational functions on $\mathbf{t}$ which have no pole at $\mathbf{t}=\mathbf{0}$. Put $\Xi_{\mathscr{A}}^{n, \mathbf{d}}=\Xi^{n, \mathbf{d}}[\mathbf{t}] \otimes_{\mathbf{Q}[\mathbf{t}]} \mathscr{A}$, and define an $\mathscr{A}$-submodule $\Xi_{\mathscr{A}}^{n, D}$ of $\Xi_{\mathbf{Q}}^{n, D}(\mathbf{t})$ by $\Xi_{\mathscr{A}}^{n, D}=\bigoplus_{\mathbf{d} \in D} \Xi_{\mathscr{A}}^{n, \mathbf{d}}$. By the specialization $\mathbf{t} \mapsto \mathbf{0}$, we have a natural map $\Xi_{\mathscr{A}}^{n, D} \rightarrow \Xi_{\mathbf{Q}}^{n, D}$. Under this specialization, the bilinear form $\langle$,$\rangle on \Xi_{\mathscr{A}}^{n, D}$ gives rise to a bilinear form on $\Xi_{\mathbf{Q}}^{n, D}$, which coincides with $\langle,\rangle_{0}$ by $(3.4 .3)$.

3.5. We consider a total order $\Lambda \preceq \Lambda^{\prime}$ on $Z_{n, D}$ satisfying the following properties;

(i) $\Lambda \prec \Lambda^{\prime}$ if $a(\Lambda)>a\left(\Lambda^{\prime}\right)$.

(ii) Each similarity class in $Z_{n, D}$ gives rise to an interval with respect to the total order $\preceq$ on $Z_{n, D}$.

In the discussion below, we denote by $\Lambda \sim \Lambda^{\prime}$ if $\Lambda, \Lambda^{\prime} \in Z_{n, D}$ belong to the same similarity class.

We can define Hall-Littlewood functions by making use of the total order $\preceq$ and the bilinear form $\langle$,$\rangle on \Xi_{\mathbf{Q}}^{n, D}(\mathbf{t})$.

Proposition 3.6. For each $\Lambda \in Z_{n, D}$ and a signature \pm , there exists a unique $P_{\Lambda}^{ \pm}(x ; \mathbf{t}) \in$ $\Xi_{\mathscr{A}}^{n, D}$ satisfying the following properties;

(i) $P_{\Lambda}^{ \pm}(x ; \mathbf{t})$ can be expressed as

$$
P_{\Lambda}^{ \pm}(x ; \mathbf{t})=s_{\Lambda}(x)+\sum_{\Lambda^{\prime} \prec \Lambda} u_{\Lambda, \Lambda^{\prime}}^{ \pm}(\mathbf{t}) s_{\Lambda^{\prime}}(x)
$$

where $u_{\Lambda, \Lambda^{\prime}}^{ \pm}(\mathbf{t}) \in \mathscr{A}$, and $u_{\Lambda, \Lambda^{\prime}}^{ \pm}(\mathbf{t})=0$ if $\Lambda^{\prime} \sim \Lambda$.

(ii) $\left\langle P_{\Lambda}^{+}, P_{\Lambda^{\prime}}^{-}\right\rangle=0$ if $\Lambda \nsim \Lambda^{\prime}$.

(iii) $P_{\Lambda}^{ \pm}(x ; \mathbf{0})=s_{\Lambda}(x)$.

Proof. We shall construct the functions $P_{\Lambda}^{ \pm}(x ; \mathbf{t})$ satisfying the properties (i) $\sim$ (iii) by induction on the total order $\preceq$. Let $\Lambda_{0}$ be the minimum element with respect to $\preceq$. By (i), $P_{\Lambda_{0}}^{ \pm}$must coincide with $s_{\Lambda_{0}}$. Moreover by (i), $P_{\Lambda}^{ \pm}=s_{\Lambda}$ if $\Lambda \sim \Lambda_{0}$. These functions satisfy the conditions (i), (ii) and (iii). Hence $P_{\Lambda}^{ \pm}$can be constructed for the similarity class containing $\Lambda_{0}$. Now take $\Lambda \in Z_{n, D}$, and assume, for $\Lambda^{\prime} \prec \Lambda, \Lambda^{\prime \prime} \prec \Lambda$, that $P_{\Lambda^{\prime}}^{+}, P_{\Lambda^{\prime \prime}}^{-}$ are constructed, where they satisfy the properties (i), (iii) and

$$
(\text { ii) })^{\prime}:\left\langle P_{\Lambda^{\prime}}^{+}, P_{\Lambda^{\prime \prime}}^{-}\right\rangle=0 \text { for } \Lambda^{\prime} \nsim \Lambda^{\prime \prime} \text {. }
$$


We note, under our assumption, that the condition (i) is equivalent to the condition

$$
P_{\Lambda}^{ \pm}=s_{\Lambda}+\sum_{\Lambda^{\prime} \prec \Lambda} d_{\Lambda, \Lambda^{\prime}}^{ \pm} P_{\Lambda^{\prime}}^{ \pm}
$$

where $d_{\Lambda, \Lambda^{\prime}}^{ \pm} \in \mathscr{A}$ and $d_{\Lambda, \Lambda^{\prime}}^{ \pm}=0$ if $\Lambda^{\prime} \sim \Lambda$. If we consider the scalar product with $P_{\Lambda^{\prime \prime}}^{-}$on both sides of (3.6.2), we have, by (ii)',

$$
\left\langle P_{\Lambda}^{+}, P_{\Lambda^{\prime \prime}}^{-}\right\rangle=\left\langle s_{\Lambda}, P_{\Lambda^{\prime \prime}}^{-}\right\rangle+\sum_{\Lambda^{\prime} \sim \Lambda^{\prime \prime}} d_{\Lambda, \Lambda^{\prime}}^{+}\left\langle P_{\Lambda^{\prime}}^{+}, P_{\Lambda^{\prime \prime}}^{-}\right\rangle
$$

By (iii) and the discussion in 3.4, we see that $\left.\left\langle P_{\Lambda^{\prime}}^{+}, P_{\Lambda^{\prime \prime}}^{-}\right\rangle\right|_{\mathbf{t}=\mathbf{0}}=\left\langle s_{\Lambda^{\prime}}, s_{\Lambda^{\prime \prime}}\right\rangle_{0}=\delta_{\Lambda^{\prime}, \Lambda^{\prime \prime}}$. In particular, if we put $\mathscr{C}$ the similarity class containing $\Lambda^{\prime \prime}$, the matrix $B_{\mathscr{C}}=\left(\left\langle P_{\Lambda^{\prime}}^{+}, P_{\Lambda^{\prime \prime}}^{-}\right\rangle\right)_{\Lambda^{\prime}, \Lambda^{\prime \prime} \in \mathscr{C}}$ satisfies the property that $\left(\operatorname{det} B_{\mathscr{C}}\right)_{\mathbf{t}=\mathbf{0}} \neq 0$. It follows that the system of linear equations with respect to the unknown variables $u_{\Lambda^{\prime}}\left(\Lambda^{\prime} \in \mathscr{C}\right)$

$$
\left\langle s_{\Lambda}, P_{\Lambda^{\prime \prime}}^{-}\right\rangle+\sum_{\Lambda^{\prime} \in \mathscr{C}} u_{\Lambda^{\prime}}\left\langle P_{\Lambda^{\prime}}^{+}, P_{\Lambda^{\prime \prime}}^{-}\right\rangle=0
$$

has a unique solution $u_{\Lambda^{\prime}}=d_{\Lambda, \Lambda^{\prime}}^{+}$. Since $\left(\operatorname{det} B_{\mathscr{C}}\right)_{\mathbf{t}=\mathbf{0}} \neq 0$, we have $d_{\Lambda, \Lambda^{\prime}}^{+} \in \mathscr{A}$. We now define $d_{\Lambda, \Lambda^{\prime}}^{+}$as above for any similarity class $\mathscr{C}$ containing $\Lambda^{\prime \prime}$ such that $\Lambda^{\prime \prime} \prec \Lambda, \Lambda^{\prime \prime} \nsim \Lambda$, and define $P_{\Lambda}^{+}$by using the formula (3.6.2). Then for any $\Lambda^{\prime} \prec \Lambda, \Lambda^{\prime} \nsim \Lambda$, we have $\left\langle P_{\Lambda}^{+}, P_{\Lambda^{\prime}}^{-}\right\rangle=0$. Also it follows from the construction that $d_{\Lambda, \Lambda^{\prime}}^{+}=0$ for $\Lambda^{\prime} \sim \Lambda$. On the other hand, if we put $\mathbf{t}=\mathbf{0}$ in (3.6.4), we have $d_{\Lambda, \Lambda^{\prime}}^{+}(\mathbf{0})=0$ since $\left.\left\langle s_{\Lambda}, P_{\Lambda^{\prime \prime}}^{-}\right\rangle\right|_{\mathbf{t}=\mathbf{0}}=\left\langle s_{\Lambda}, s_{\Lambda^{\prime \prime}}\right\rangle_{0}=0$. Hence $P_{\Lambda}^{+}(x ; \mathbf{0})=s_{\Lambda}(x)$. Thus $P_{\Lambda}^{+}$satisfies the condition (i), (iii) and (ii)'.

Instead of (3.6.3), if we compute $\left\langle P_{\Lambda^{\prime}}^{+}, P_{\Lambda}^{-}\right\rangle$, in a similar way as above we can construct $P_{\Lambda}^{-}$satisfying (i), (iii) and (ii) $)^{\prime}$. Thus $P_{\Lambda}^{ \pm}$is constructed.

Next we show the uniqueness. By induction, we may assume that $P_{\Lambda^{\prime}}^{ \pm}$is already determined uniquely for any $\Lambda^{\prime} \prec \Lambda$. Suppose that both of $P_{\Lambda}^{+}, P_{\Lambda}^{\prime+}$ satisfy the condition (i) and (ii). Then by (i),

$$
X=P_{\Lambda}^{+}-P_{\Lambda}^{\prime+}=\sum_{\substack{\Lambda^{\prime} \prec \Lambda \\ \Lambda^{\prime} \not \Lambda}} b_{\Lambda^{\prime}} P_{\Lambda^{\prime}}^{+}
$$

Hence $\left\langle X, P_{\Lambda^{\prime}}^{-}\right\rangle=0$ for $\Lambda^{\prime} \succ \Lambda$ or $\Lambda^{\prime} \sim \Lambda$. On the other hand, for $\Lambda^{\prime} \prec \Lambda, \Lambda^{\prime} \nsim \Lambda$, we have $\left\langle P_{\Lambda}^{+}, P_{\Lambda^{\prime}}^{-}\right\rangle=0,\left\langle P_{\Lambda}^{\prime+}, P_{\Lambda^{\prime}}^{-}\right\rangle=\left\langle P_{\Lambda}^{\prime+}, P_{\Lambda^{\prime}}^{\prime-}\right\rangle=0$. Hence $\left\langle X, P_{\Lambda^{\prime}}^{-}\right\rangle=0$. It follows that $\left\langle X, P_{\Lambda^{\prime}}^{-}\right\rangle=0$ for any $\Lambda^{\prime}$. Since $\left\{P_{\Lambda^{\prime}}^{-} \mid \Lambda^{\prime} \in Z_{n, D}\right\}$ is a basis of $\Xi_{\mathbf{Q}}^{n, D}(\mathbf{t})$, and $\langle$,$\rangle is non-degenerate, we$ see that $X=0$, namely $P_{\Lambda}^{+}=P_{\Lambda}^{\prime+}$. The uniqueness of $P_{\Lambda}^{-}$can be shown similarly. The proposition is proved.

3.7. It follows from Proposition 3.6 (i), that $\left\{P_{\Lambda}^{ \pm} \mid \Lambda \in Z_{n, D}\right\}$ gives rise to an $\mathscr{A}$-basis of $\Xi_{\mathscr{A}}^{n, D}$. Let $\mathscr{C}$ be a similarity class in $Z_{n, D}$. The proof of Proposition 3.6 shows that $B=\left(\left\langle P_{\Lambda}^{+}, P_{\Lambda^{\prime}}^{-}\right\rangle\right)_{\Lambda, \Lambda^{\prime} \in \mathscr{C}}$ is non-singular and $\left.(\operatorname{det} B)\right|_{\mathbf{t}=\mathbf{0}} \neq 0$. Hence if we put $B^{-1}=B_{\mathscr{C}}=\left(b_{\Lambda, \Lambda^{\prime}}(\mathbf{t})\right)$, then $b_{\Lambda, \Lambda^{\prime}}(\mathbf{t}) \in \mathscr{A}$. We now define symmetric functions 
$Q_{\Lambda}^{ \pm}(x ; \mathbf{t}) \in \Xi_{\mathscr{A}}^{n, D}$ by

$$
\begin{aligned}
& Q_{\Lambda}^{+}(x ; \mathbf{t})=\sum_{\Lambda^{\prime} \sim \Lambda} b_{\Lambda, \Lambda^{\prime}}(\mathbf{t}) P_{\Lambda^{\prime}}^{+}(x ; \mathbf{t}), \\
& Q_{\Lambda}^{-}(x ; \mathbf{t})=\sum_{\Lambda^{\prime} \sim \Lambda} b_{\Lambda^{\prime}, \Lambda}(\mathbf{t}) P_{\Lambda^{\prime}}^{-}(x ; \mathbf{t}) .
\end{aligned}
$$

Then $\left\{Q_{\Lambda}^{ \pm} \mid \Lambda \in Z_{n, D}\right\}$ gives rise to an $\mathscr{A}$-basis of $\Xi_{\mathscr{A}}^{n, D} . P_{\Lambda}^{ \pm}$and $Q_{\Lambda}^{ \pm}$are called HallLittlewood functions attached to $r$-symbols.

The orthogonality relations for $P_{\Lambda}^{ \pm}$in Proposition 3.6 (ii) and the definition of $Q_{\Lambda}^{ \pm}$ imply that

$$
\left\langle P_{\Lambda}^{+}, Q_{\Lambda^{\prime}}^{-}\right\rangle=\left\langle Q_{\Lambda}^{+}, P_{\Lambda^{\prime}}^{-}\right\rangle=\delta_{\Lambda, \Lambda^{\prime}}
$$

Here we put

$$
\Omega^{n, D}(x, y ; \mathbf{t})=\sum_{\mathbf{d} \in D} \Omega^{n, \mathbf{d}}(x, y ; \mathbf{t})
$$

Then (3.2.1) can be rewritten as

$$
\Omega^{n, D}(x, y ; \mathbf{t})=\sum_{\Lambda, \Lambda^{\prime} \in Z_{n, D}} z_{\Lambda, \Lambda^{\prime}}(\mathbf{t}) p_{\Lambda}(x) \bar{p}_{\Lambda}(y),
$$

where we put $z_{\Lambda, \Lambda^{\prime}}(\mathbf{t})=0$ if $d(\Lambda) \neq d\left(\Lambda^{\prime}\right)$. Now the orthogonality relations (3.7.2) can be rewritten as follows:

Lemma 3.8. Under the notation above,

$$
\begin{aligned}
& \Omega^{n, D}(x, y ; \mathbf{t})=\sum_{\Lambda, \Lambda^{\prime}} b_{\Lambda^{\prime}, \Lambda}(\mathbf{t}) P_{\Lambda}^{+}(x ; \mathbf{t}) P_{\Lambda^{\prime}}^{-}(y ; \mathbf{t}), \\
& \Omega^{n, D}(x, y ; \mathbf{t})=\sum_{\Lambda} P_{\Lambda}^{+}(x ; \mathbf{t}) Q_{\Lambda}^{-}(y ; \mathbf{t})=\sum_{\Lambda} Q_{\Lambda}^{+}(x ; \mathbf{t}) P_{\Lambda}^{-}(y ; \mathbf{t}),
\end{aligned}
$$

where $\Lambda, \Lambda^{\prime}$ run over all elements in $Z_{n, D}$. We put $b_{\Lambda, \Lambda^{\prime}}(t)=0$ unless $\Lambda \sim \Lambda^{\prime}$.

Proof. Since $P_{\Lambda}^{+}$and $Q_{\Lambda^{\prime}}^{-}, Q_{\Lambda}^{+}$and $P_{\Lambda^{\prime}}^{-}$, are dual basis with respect to the form $\langle\rangle,,(3.8 .2)$ is obtained by a similar argument as in [Mac, Chap. I,4]. Then by using (3.7.1), we obtain $(3.8 .1)$.

The following result gives a characterization of Hall-Littlewood functions $P_{\Lambda}^{ \pm}$and $Q_{\Lambda}^{ \pm}$.

Theorem 3.9. $P_{\Lambda}^{ \pm}(x ; \mathbf{t})$ is determined uniquely as the function satisfying the following properties. Let $\mathscr{C}$ be the similarity class containing $\Lambda$.

(i) $P_{\Lambda}^{ \pm}(x ; \mathbf{t})$ can be written as

$$
P_{\Lambda}^{ \pm}(x ; \mathbf{t})=\sum_{(*)} c_{\Lambda, \Lambda^{\prime}}(\mathbf{t}) q_{\Lambda^{\prime}}^{ \pm}(x ; \mathbf{t})
$$


where $\left(^{*}\right)$ is that $\Lambda^{\prime}$ runs over all elements in $Z_{n, D}$ such that $\Lambda^{\prime} \sim \Lambda$ or $\Lambda^{\prime} \succeq \Lambda$. Moreover $c_{\Lambda, \Lambda^{\prime}}(\mathbf{t}) \in \mathbf{Q}(\mathbf{t})$, and $c(\mathbf{t})_{\mathscr{C}}=\left(c_{\Lambda^{\prime}, \Lambda^{\prime \prime}}(\mathbf{t})\right)_{\Lambda^{\prime}, \Lambda^{\prime \prime} \in \mathscr{C}}$ is a non-singular matrix. (ii) $P_{\Lambda}^{ \pm}(x ; \mathbf{t})$ can be written as

$$
P_{\Lambda}^{ \pm}(x ; \mathbf{t})=\sum_{(* *)} u_{\Lambda, \Lambda^{\prime}}(\mathbf{t}) s_{\Lambda^{\prime}}(x)
$$

where $\left({ }^{* *}\right)$ is that $\Lambda^{\prime}$ runs over all elements in $Z_{n, D}$ such that $\Lambda^{\prime} \sim \Lambda$ or $\Lambda^{\prime} \preceq \Lambda$. Moreover $u_{\Lambda, \Lambda^{\prime}}(\mathbf{t}) \in \mathbf{Q}(\mathbf{t})$, and $u(\mathbf{t})_{\mathscr{C}}=\left(u_{\Lambda^{\prime}, \Lambda^{\prime \prime}}(\mathbf{t})\right)_{\Lambda^{\prime}, \Lambda^{\prime \prime} \in \mathscr{C}}$ is the identity matrix.

Similarly, $Q_{\Lambda}^{ \pm}(x ; \mathbf{t})$ is determined uniquely by similar formulas as in (3.9.1) and (3.9.2), where the conditions for $c(\mathbf{t})_{\mathscr{C}}$ and $u(\mathbf{t})_{\mathscr{C}}$ are interchanged so that $c(\mathbf{t})_{\mathscr{C}}$ is the identity matrix, and $u(\mathbf{t})_{\mathscr{C}}$ is a non-singular matrix.

Proof. For two bases $u=\left\{u_{\Lambda}\right\}, v=\left\{v_{\Lambda}\right\}$ of $\Xi_{\mathbf{Q}}^{n, D}(\mathbf{t})$, we denote by $M=M(u, v)$ the transition matrix $\left(m_{\Lambda, \Lambda^{\prime}}\right)$ of those two bases, namely $u_{\Lambda}=\sum_{\Lambda^{\prime}} m_{\Lambda, \Lambda^{\prime}} v_{\Lambda^{\prime}}$. We consider the following bases of $\Xi_{\mathbf{Q}}^{n, D}(\mathbf{t}), P^{ \pm}=\left\{P_{\Lambda}^{ \pm}\right\}, q^{ \pm}=\left\{q_{\Lambda}^{ \pm}\right\}, s=\left\{s_{\Lambda}\right\}$, and $m=\left\{m_{\Lambda}\right\}$. Put

$$
A_{ \pm}=M\left(q^{ \pm}, P^{ \pm}\right), \quad B_{ \pm}=M\left(m, P^{ \pm}\right) .
$$

In the following discussion, we consider the matrices indexed by $Z_{n, D}$ as the block matrices with respect to the partition of $Z_{n, D}$ arising from similarity classes. We show that $A_{ \pm}$is upper triangular as a block matrix. By Proposition 3.6, $M\left(P^{ \pm}, s\right)$ is a lower triangular block matrix, and the diagonal block is the identity matrix. On the other hand, we note that $M(s, m)$ is a lower uni-triangular block matrix. In fact, for $\boldsymbol{\lambda}, \boldsymbol{\mu} \in \mathscr{P}_{n^{\prime}, r}, s_{\boldsymbol{\lambda}}$ is written as a linear combination of $m_{\boldsymbol{\mu}}$. Moreover, if we write $\boldsymbol{\lambda}=\left(\lambda^{(1)}, \ldots, \lambda^{(r)}\right)$, $\boldsymbol{\mu}=\left(\mu^{(1)}, \ldots, \mu^{(r)}\right)$, then $\left|\lambda^{(k)}\right|=\left|\mu^{(k)}\right|$ and $\mu^{(k)} \leq \lambda^{(k)}$ for any $k$, where the coefficient of $m_{\boldsymbol{\lambda}}$ equals 1 . By applying $\Theta_{\mathbf{d}}$, we see that $s_{\Lambda}$ is a linear combination of $m_{\Lambda^{\prime}}$, where if we write $\Lambda=\left(A^{(1)}, \ldots, A^{(r)}\right), \Lambda^{\prime}=\left(B^{(1)}, \ldots, B^{(r)}\right)$, then $A^{(k)}, B^{(k)}$ are partitions such that $\left|A^{(k)}\right|=\left|B^{(k)}\right|$ and that $B^{(k)}<A^{(k)}$ unless $B^{(k)}=A^{(k)}$ for each $k$. It follows, by using [S2, Lemma 3.9], that $a\left(\Lambda^{\prime}\right) \geq a(\Lambda)$ and the equality holds only when $\Lambda^{\prime} \sim \Lambda$. Hence, by our assumption 3.5 , we have $\Lambda \preceq \Lambda^{\prime}$ with equality only when $\Lambda^{\prime} \sim \Lambda$. This implies that $M(s, m)$ is lower uni-triangular.

From the above discussion, we see that $B_{ \pm}^{-1}=M\left(P^{ \pm}, s\right) M(s, m)$ is lower uni-triangular block matrix, and so is $B_{ \pm}$. Here put $D_{ \pm}={ }^{t} B_{\mp} A_{ \pm}$. If we write $A_{+}=\left(A_{\Lambda, \Lambda^{\prime}}^{+}\right)$, $B_{-}=\left(B_{\Lambda, \Lambda^{\prime}}^{-}\right)$, we have

$$
\begin{aligned}
\sum_{\Lambda \in Z_{n, D}} q_{\Lambda}^{+}(x ; \mathbf{t}) m_{\Lambda}(y) & =\sum_{\Lambda, \Lambda^{\prime}, \Lambda^{\prime \prime}} A_{\Lambda, \Lambda^{\prime}}^{+} B_{\Lambda, \Lambda^{\prime \prime}}^{-} P_{\Lambda^{\prime}}^{+}(x ; \mathbf{t}) P_{\Lambda^{\prime \prime}}^{-}(y ; \mathbf{t}) \\
& =\sum_{\Lambda^{\prime}, \Lambda^{\prime \prime}} b_{\Lambda^{\prime \prime}, \Lambda^{\prime}} P_{\Lambda^{\prime}}^{+}(x ; \mathbf{t}) P_{\Lambda^{\prime \prime}}^{-}(y ; \mathbf{t})
\end{aligned}
$$

The second equality is obtained by comparing (3.3.1) and (3.8.1). We compare the first and the second equalities in (3.9.3). Since $P_{\Lambda^{\prime}}^{+}(x ; \mathbf{t}) P_{\Lambda^{\prime \prime}}^{-}(y ; \mathbf{t})$ are linearly independent, this implies that $D_{+}={ }^{t} B_{-} A_{+}$is a diagonal block matrix, and that the diagonal block $D_{\mathscr{C}}^{+}$ corresponding to the similarity class $\mathscr{C}$ coincides with $B_{\mathscr{C}}=\left(b_{\Lambda^{\prime}, \Lambda^{\prime \prime}}\right)$. Hence $A_{+}$is an upper triangular block matrix, and the diagonal block corresponding to $\mathscr{C}$ coincides with 
$B_{\mathscr{C}}$. By applying a similar argument to the second identity in (3.3.1), we obtain that $A_{-}$ is an upper triangular block matrix, and the diagonal block corresponding to $\mathscr{C}$ coincides with ${ }^{t} B_{\mathscr{C}}$. This shows that $P_{\Lambda}^{ \pm}(x ; \mathbf{t})$ satisfies the relation in (i). The fact that $P_{\Lambda}^{ \pm}$satisfies the relation (ii) was already verified in Proposition 3.6.

We show the uniqueness of $P_{\Lambda}^{ \pm}$. Assume that $R(x ; \mathbf{t})$ satisfies the condition (i) and (ii) as above, replacing $P_{\Lambda}^{ \pm}$by $R$. Since $P_{\Lambda}^{ \pm}$satisfies (i) and (ii), one can write as

$$
\begin{aligned}
& s_{\Lambda^{\prime}}=\sum_{(*)} u_{\Lambda^{\prime}, \Lambda^{\prime \prime}}^{\prime} P_{\Lambda^{\prime \prime}}^{+}, \\
& q_{\Lambda^{\prime}}^{+}=\sum_{(* *)} c_{\Lambda^{\prime}, \Lambda^{\prime \prime}}^{\prime} P_{\Lambda^{\prime \prime}}^{+},
\end{aligned}
$$

where $(*)$ and $\left({ }^{*}\right)$ are similar conditions as $(*),(* *)$ in (3.9.1) and (3.9.2), by replacing $\Lambda$ by $\Lambda^{\prime}$. By substituting these equations into the expression of $R$, we have

$$
R=\sum_{(1)} u_{\Lambda, \Lambda^{\prime \prime}}^{\prime \prime} P_{\Lambda^{\prime \prime}}^{+}=\sum_{(2)} c_{\Lambda, \Lambda^{\prime \prime}}^{\prime \prime} P_{\Lambda^{\prime \prime}}^{+}
$$

where in (1) $\Lambda^{\prime \prime}$ runs over all the elements such that $\Lambda^{\prime \prime} \sim \Lambda$ or $\Lambda^{\prime \prime} \prec \Lambda$, and in (2) $\Lambda^{\prime \prime}$ runs over all the elements such that $\Lambda^{\prime \prime} \sim \Lambda$ or $\Lambda^{\prime \prime} \succeq \Lambda$. Moreover, $u^{\prime \prime}(\mathbf{t})_{\mathscr{C}}=\left(u_{\Lambda^{\prime}, \Lambda^{\prime \prime}}^{\prime \prime}(\mathbf{t})\right)$ is the identity matrix. It follows that $R=P_{\Lambda}^{+}$. The proof for $P_{\Lambda}^{-}$is similar. Thus the uniqueness of $P_{\Lambda}^{ \pm}$is proved.

Let $c_{\Lambda, \Lambda^{\prime}}^{ \pm}(\mathbf{t})$ be the coefficient $c_{\Lambda, \Lambda^{\prime}}(\mathbf{t})$ for $P_{\Lambda}^{ \pm}(x ; \mathbf{t})$ in $(3.9 .1)$. It follows from the proof of (i) in the theorem, that $\left(c^{+}(\mathbf{t})\right)_{\mathscr{C}}=B_{\mathscr{C}}^{-1},\left(c^{-}(\mathbf{t})\right)_{\mathscr{C}}={ }^{t} B_{\mathscr{C}}^{-1}$. Hence we obtain the formulas for $Q_{\Lambda}^{+}$if we multiply $B_{\mathscr{C}}$ on the both sides of (3.9.1) and (3.9.2) with respect to $P_{\Lambda}^{+}$. Similarly, we obtain the formulas for $Q_{\Lambda}^{-}$if we multiply ${ }^{t} B_{\mathscr{C}}$ on the both sides of (3.9.1) and (3.9.2) with respect to $P_{\Lambda}^{-}$. Thus we obtain the assertion for $Q_{\Lambda}^{ \pm}$. The theorem is proved.

3.10. Since $\left\{P_{\Lambda}^{ \pm} \mid \Lambda \in Z_{n, D}\right\}$ and $\left\{s_{\Lambda} \mid \Lambda \in Z_{n, D}\right\}$ give bases of $\Xi_{\mathbf{Q}}^{n, D}(\mathbf{t})$, there exist unique functions $K_{\Lambda, \Lambda^{\prime}}^{ \pm}(\mathbf{t}) \in \mathbf{Q}(\mathbf{t})$ satisfying the formula;

$$
s_{\Lambda}(x)=\sum_{\Lambda^{\prime} \in Z_{n, D}} K_{\Lambda, \Lambda^{\prime}}^{ \pm}(\mathbf{t}) P_{\Lambda^{\prime}}^{ \pm}(x ; \mathbf{t})
$$

$K_{\Lambda, \Lambda^{\prime}}^{ \pm}(\mathbf{t})$ is called the Kostka function associated to complex reflection groups, or associated to $r$-symbols, in short. It is known that the original Hall-Littlewood functions $P_{\lambda}$ associated to partitions $\lambda$ have similar properties as in Theorem 3.9. Hence by the uniqueness, our Kostka function coincides with Kostka polynomial in the case where $r=1, e=0, \mathbf{s}=\mathbf{0}$. In the case where $r=2$, we have $K_{\Lambda, \Lambda^{\prime}}^{+}(\mathbf{t})=K_{\Lambda, \Lambda^{\prime}}^{-}(\mathbf{t})$ since $q_{\Lambda}^{+}=q_{\Lambda}^{-}$and so $P_{\Lambda}^{+}=P_{\Lambda}^{-}$. In this case, we denote $K_{\Lambda, \Lambda^{\prime}}^{ \pm}(\mathbf{t})$ as $K_{\Lambda, \Lambda^{\prime}}(\mathbf{t})$.

The following properties are easily deduced from Theorem 3.9 (ii).

$$
K_{\Lambda, \Lambda}^{ \pm}(\mathbf{t})=1, K_{\Lambda, \Lambda^{\prime}}^{ \pm}(\mathbf{t})=0 \text { for } \Lambda^{\prime} \sim \Lambda, \Lambda^{\prime} \neq \Lambda \text {. Moreover, } K_{\Lambda, \Lambda^{\prime}}^{ \pm}(\mathbf{t})=0 \text { if } \Lambda^{\prime} \succ \Lambda .
$$

In the one-parameter case, we define a modified Kostka function $\widetilde{K}_{\Lambda, \Lambda^{\prime}}^{ \pm}(t) \in \mathbf{Q}(t)$ by 


$$
\widetilde{K}_{\Lambda, \Lambda^{\prime}}^{ \pm}(t)=t^{a\left(\Lambda^{\prime}\right)} K_{\Lambda, \Lambda^{\prime}}^{ \pm}\left(t^{-1}\right)
$$

Remarks 3.11. (i) Our definition of Hall-Littlewood functions and Kostka functions depend on the choice of a total order $\preceq$ on $Z_{n, D}$. It is not known, in general, whether or not those functions actually depend on the total order. Also our Kostka function $K_{\Lambda, \Lambda^{\prime}}^{ \pm}(\mathbf{t})$ are rational functions on $\mathbf{t}$. It is not known in general whether $K_{\Lambda, \Lambda^{\prime}}^{ \pm}(\mathbf{t}) \in \mathbf{Z}[\mathbf{t}]$ or not.

(ii) In [S4], the notion of limit symbols was introduced, which is defined by a special choice of $e$ and $\mathbf{s}$ so that each similarity class consists of a single element. In that case (for the multi-parameter, with $D=\left\{d^{\bullet}\right\}$ ), it is proved in [S6] that $P_{\Lambda}^{ \pm}(x ; \mathbf{t}) \in \Xi^{n, \mathbf{d}^{\bullet}}[\mathbf{t}]$ and that $K_{\Lambda, \Lambda^{\prime}}^{ \pm}(\mathbf{t}) \in \mathbf{Z}[\mathbf{t}]$. Moreover, those functions do not depend on the choice of the total order whenever it is compatible with the dominance order on $\mathscr{P}_{n, r}$.

(iii) In the limit symbol case, it is proved in [S6] that our Kostka function $K_{\Lambda, \Lambda^{\prime}}^{-}(\mathbf{t})$ coincides with the function introduced by Finkelberg-Ionov [FI]. By using their results, it is shown that $K_{\Lambda, \Lambda^{\prime}}^{-}(\mathbf{t})$ has a geometric realization in terms of coherent sheaves, which implies that $K_{\Lambda, \Lambda^{\prime}}^{-}(\mathbf{t})$ is a polynomial in $t_{1}, \ldots, t_{r}$ with non-negative coefficients.

\section{Algorithm of computing Kostka functions}

4.1. In [S2], it was shown that there exists an algorithm of computing Kostka functions associated to $Z_{n, D}$ with $D=\left\{\mathbf{d}^{\bullet}\right\}$. In [S6], multi-parameter Kostka functions associated to limit symbols were introduced, but the algorithm of computing them was not discussed there. In this section, we show that a similar algorithm as in [S2] holds also for our Kostka functions (with multi-parameter) associated to $Z_{n, D}$ for any $D$.

Let $W_{n, r}$ be the complex reflection group $S_{n} \ltimes(\mathbf{Z} / r \mathbf{Z})^{n}$. It is known that the irreducible characters of $W_{n, r}$ are parametrized by $\mathscr{P}_{n, r}$. We denote by $\chi^{\lambda}$ the irreducible character of $W_{n, r}$ corresponding to $\boldsymbol{\lambda} \in \mathscr{P}_{n, r}$.

4.2. We consider the matrix $X_{ \pm}(\mathbf{t})=M\left(p, P^{ \pm}\right)$, where $p=\left\{p_{\Lambda}\right\}$ is the basis of $\Xi_{\mathbf{C}}^{n, D}(\mathbf{t})$ consisting of power sum symmetric functions, and $P^{ \pm}=\left\{P_{\Lambda}^{ \pm}\right\}$are bases of $\Xi_{\mathbf{Q}}^{n, D}(\mathbf{t})$ consisting of Hall-Littlewood functions. Thus $X_{ \pm}(\mathbf{t})=\left(X_{\Lambda, \Lambda^{\prime}}^{ \pm}(\mathbf{t})\right)$, where $X_{\Lambda, \Lambda^{\prime}}^{ \pm}(\mathbf{t}) \in \mathbf{C}(\mathbf{t})$ such that

$$
p_{\Lambda}(x)=\sum_{\Lambda^{\prime} \in Z_{n, D}} X_{\Lambda, \Lambda^{\prime}}^{ \pm}(\mathbf{t}) P_{\Lambda^{\prime}}^{ \pm}(x ; \mathbf{t})
$$

In the classical case, Green polynomials $Q_{\lambda, \mu}(t)$ are defined by $Q_{\lambda, \mu}(t)=t^{n(\mu)} X_{\lambda, \mu}\left(t^{-1}\right)$ (see [Mac, III, 7]), where $X_{\lambda, \mu}(t)$ is defined similarly to (4.2.1) with respect to $p_{\lambda}(x)$ and $P_{\mu}(x ; t)$ for $\lambda, \mu \in \mathscr{P}_{n}$. Thus we call $X_{\Lambda, \Lambda^{\prime}}^{ \pm}(\mathbf{t})$ the generalized Green functions associated to $r$-symbols.

By Proposition 3.6 (iii), we have $P_{\Lambda^{\prime}}^{ \pm}(x ; \mathbf{0})=s_{\Lambda^{\prime}}(x)$. By the Frobenius formula (see $[\mathrm{Mac}])$, we know, for $\boldsymbol{\lambda}, \boldsymbol{\mu} \in \mathscr{P}_{n^{\prime}, r}$, that

$$
p_{\boldsymbol{\lambda}}=\sum_{\boldsymbol{\mu} \in \mathscr{P}_{n^{\prime}, r}} \chi^{\boldsymbol{\mu}}\left(w_{\boldsymbol{\lambda}}\right) s_{\boldsymbol{\mu}}
$$


where $w_{\boldsymbol{\lambda}}$ is a representative of the conjugacy class in $W_{n^{\prime}, r}$ corresponding to $\boldsymbol{\lambda} \in \mathscr{P}_{n^{\prime}, r}$. (If $w \in W_{n^{\prime}, r}$ is conjugate to $w_{\boldsymbol{\lambda}}$, we say that $w$ has type $\boldsymbol{\lambda}$ ). Hence by the construction of $p_{\Lambda}$ and $s_{\Lambda^{\prime}}$ in terms of the isomorphism $\Theta_{\mathbf{d}}$, we have the following.

(4.2.2) Assume that $\Lambda, \Lambda^{\prime} \in Z_{n, D}$. If $d(\Lambda) \neq d\left(\Lambda^{\prime}\right)$, then $X_{\Lambda, \Lambda^{\prime}}^{ \pm}(\mathbf{0})=0$. If $d(\Lambda)=d\left(\Lambda^{\prime}\right)=\mathbf{d}$ and $\Lambda=\Lambda(\boldsymbol{\lambda}), \Lambda^{\prime}=\Lambda(\boldsymbol{\mu})$ with $\boldsymbol{\lambda}, \boldsymbol{\mu} \in \mathscr{P}_{n^{\prime}, r}$ (under the isomorphism $\Theta_{\mathbf{d}}: \widehat{\Xi}_{\mathbf{Q}}^{n^{\prime}, \mathbf{0}}(\mathbf{t}) \simeq$ $\left.\Xi_{\mathbf{Q}}^{n, \mathbf{d}}(\mathbf{t})\right)$, then

$$
X_{\Lambda, \Lambda^{\prime}}^{ \pm}(\mathbf{0})=\chi^{\boldsymbol{\mu}}\left(w_{\boldsymbol{\lambda}}\right)
$$

In particular, $X_{ \pm}(\mathbf{0})$ (after a suitable permutation of indices) turns out to be a diagonal block matrix with respect to the partition $Z_{n, D}=\coprod_{\mathbf{d}} Z_{n, \mathbf{d}}$, and the diagonal block corresponding to $Z_{n, \mathbf{d}}$ is exactly the character table of $W_{n^{\prime}, r}$. Since $X_{ \pm}(\mathbf{0})$ does not depend on the signature, we denote it by $X(\mathbf{0})$.

On the other hand, by (3.7.4) and (3.8.1), we have

$$
\sum_{\Lambda, \Lambda^{\prime} \in Z_{n, D}} z_{\Lambda, \Lambda^{\prime}}(\mathbf{t}) p_{\Lambda}(x) \bar{p}_{\Lambda}(y)=\sum_{\Lambda, \Lambda^{\prime} \in Z_{n, D}} b_{\Lambda^{\prime}, \Lambda}(\mathbf{t}) P_{\Lambda}^{+}(x ; \mathbf{t}) P_{\Lambda^{\prime}}^{-}(y ; \mathbf{t}) .
$$

Put $D(\mathbf{t})=\left(b_{\Lambda^{\prime}, \Lambda}(\mathbf{t})\right)$. Then $D(\mathbf{t})$ is a diagonal block matrix with respect to the similarity classes in $Z_{n, D}$. Put $Z(\mathbf{t})=\left(z_{\Lambda, \Lambda^{\prime}}(\mathbf{t})\right) . Z(\mathbf{t})$ is a non-singular matrix since $Z\left(\mathbf{t}_{0}\right)$ is non-singular by Remark 2.7. It followed from the definition that

$$
Z(\mathbf{t}) \text { is a block diagonal matrix with respect to the partition } Z_{n, D}=\coprod_{\mathbf{d}} Z_{n, \mathbf{d}} \text {. }
$$

By substituting the formula for $p_{\Lambda}(x)$ in (4.2.1) into the left hand side of (4.2.3), and by comparing the coefficients of $P_{\Lambda}^{+}(x ; \mathbf{t}) P_{\Lambda^{\prime}}^{-}(y ; \mathbf{t})$, we obtain the following equation of matrices.

$$
{ }^{t} X_{+}(\mathbf{t}) Z(\mathbf{t}) \bar{X}_{-}(\mathbf{t})=D(\mathbf{t})
$$

where $\bar{X}_{-}(\mathbf{t})$ denotes the complex conjugate of the matrix $X_{-}(\mathbf{t})$. (Note that $P_{\Lambda}^{ \pm}(x ; \mathbf{t}) \in$ $\Xi_{\mathbf{Q}}^{n, D}(\mathbf{t})$.) If we put $\Lambda(\mathbf{t})=D(\mathbf{t})^{-1},(4.2 .5)$ can be rewritten as

$$
\bar{X}_{-}(\mathbf{t}) \Lambda(\mathbf{t})^{t} X_{+}(\mathbf{t})=Z(\mathbf{t})^{-1}
$$

Let $K_{ \pm}(\mathbf{t})=M\left(s, P^{ \pm}\right)$be the transition matrix between Schur functions and HallLittlewood functions, namely,

$$
s_{\Lambda}(x)=\sum_{\Lambda^{\prime} \in Z_{n, D}} K_{\Lambda, \Lambda^{\prime}}^{ \pm}(\mathbf{t}) P_{\Lambda^{\prime}}^{ \pm}(x ; \mathbf{t}) .
$$

By Proposition 3.6, $K_{ \pm}(\mathbf{t})$ is a lower triangular block matrix, and all the diagonal blocks are identity matrices. Moreover, $K_{\Lambda, \Lambda^{\prime}}^{ \pm}(\mathbf{t}) \in \mathbf{Q}(\mathbf{t})$, and $K_{ \pm}(\mathbf{0})$ is the identity matrix.

Since $M\left(s, P^{ \pm}\right)=M(p, s)^{-1} M\left(p, P^{ \pm}\right)$, we have $K_{ \pm}(\mathbf{t})=X(\mathbf{0})^{-1} X_{ \pm}(\mathbf{t})$. Substituting this formula into (4.2.6), we obtain

$$
K_{-}(\mathbf{t}) \Lambda(\mathbf{t})^{t} K_{+}(\mathbf{t})=\bar{X}(\mathbf{0})^{-1} Z(\mathbf{t})^{-1}{ }^{t} X(\mathbf{0})^{-1} .
$$


Put $z_{\Lambda}=z_{\boldsymbol{\lambda}}$ for $\Lambda=\Lambda(\boldsymbol{\lambda})$, and let $H$ be the diagonal matrix whose $\Lambda \Lambda$-entry is $z_{\Lambda}^{-1}$. Note that $z_{\boldsymbol{\lambda}}=\left|Z_{W^{\prime}}\left(w_{\boldsymbol{\lambda}}\right)\right|$ for $W^{\prime}=W_{n^{\prime}, r}$. Since $X(\mathbf{0})$ is the character table of $W_{n^{\prime}, r}$ on each $Z_{n, \mathbf{d}}$, by the orthogonality relations of irreducible characters of $W_{n^{\prime}, r}$, we have ${ }^{t} X(\mathbf{0}) H \bar{X}(\mathbf{0})=I$. Substituting this into (4.2.7), we have

$$
K_{-}(\mathbf{t}) \Lambda(\mathbf{t}){ }^{t} K_{+}(\mathbf{t})={ }^{t} X(\mathbf{0}) H Z(\mathbf{t})^{-1} H \bar{X}(\mathbf{0}) .
$$

4.3. Put $Z(\mathbf{t})^{-1}=\left(z_{\Lambda, \Lambda^{\prime}}^{\prime}(\mathbf{t})\right)$. Then $z_{\Lambda, \Lambda^{\prime}}^{\prime}(\mathbf{t})=0$ unless $d(\Lambda)=d\left(\Lambda^{\prime}\right)$. For $w, w^{\prime} \in$ $W_{n^{\prime}, r}$, we define $z_{w, w^{\prime}}^{\prime}(\mathbf{t})$ as follows; assume that $w$ is of type $\boldsymbol{\lambda}$ and $w^{\prime}$ is of type $\boldsymbol{\mu}$ for $\boldsymbol{\lambda}, \boldsymbol{\mu} \in \mathscr{P}_{n^{\prime}, r}$, and then put $z_{w, w^{\prime}}^{\prime}(\mathbf{t})=z_{\Lambda, \Lambda^{\prime}}^{\prime}(\mathbf{t})$ for $\Lambda=\Lambda(\boldsymbol{\lambda}), \Lambda^{\prime}=\Lambda(\boldsymbol{\mu})$.

Put $W^{\prime}=W_{n^{\prime}, r}$. For $\boldsymbol{\lambda}, \boldsymbol{\mu} \in \mathscr{P}_{n^{\prime}, r}$, we define $\omega_{\boldsymbol{\lambda}, \boldsymbol{\mu}}(\mathbf{t})$ by

$$
\omega_{\boldsymbol{\lambda}, \boldsymbol{\mu}}(\mathbf{t})=\frac{1}{\left|W^{\prime}\right|^{2}} \sum_{w, w^{\prime} \in W^{\prime}} \chi^{\boldsymbol{\lambda}}(w) \overline{\chi^{\boldsymbol{\mu}\left(w^{\prime}\right)}} z_{w, w^{\prime}}^{\prime}(\mathbf{t}) .
$$

We define a matrix $\boldsymbol{\Omega}=\left(\omega_{\Lambda, \Lambda^{\prime}}(\mathbf{t})\right)$ by

$$
\omega_{\Lambda, \Lambda^{\prime}}(\mathbf{t})= \begin{cases}\omega_{\boldsymbol{\lambda}, \boldsymbol{\mu}}(\mathbf{t}) & \text { if } \Lambda=\Lambda(\boldsymbol{\lambda}), \Lambda^{\prime}=\Lambda(\boldsymbol{\mu}) \text { with } \boldsymbol{\lambda}, \boldsymbol{\mu} \in \mathscr{P}_{n^{\prime}, r}, \\ 0 & \text { if } d(\Lambda) \neq d\left(\Lambda^{\prime}\right) .\end{cases}
$$

Note that the matrix $Z(\mathbf{t})^{-1}$ is computable by (2.6.3) and (2.9.1). Hence the matrix $\boldsymbol{\Omega}$ is computable if we know the character table of $W_{n^{\prime}, r}$ for various $n^{\prime}$.

The following result gives an algorithm of computing $K_{ \pm}(\mathbf{t})$. In the following, we consider the block matrices with respect to the similarity classes in $Z_{n, D}$.

Theorem 4.4. $\quad$ (i) The matrix $K_{ \pm}(\mathbf{t})$ is determined as the unique solution of the following equation satisfying the properties (a) and (b).

$$
\mathbf{P}_{-} \boldsymbol{\Lambda}^{t} \mathbf{P}_{+}=\boldsymbol{\Omega}
$$

(a) $\mathbf{P}_{ \pm}$is a lower triangular block matrix, where the diagonal block is the identity matrix.

(b) $\boldsymbol{\Lambda}$ is a diagonal block matrix.

Then $K_{ \pm}(\mathbf{t})=\mathbf{P}_{ \pm}$, and there exists an algorithm of computing $K_{ \pm}(\mathbf{t})$.

(ii) If $\Lambda, \Lambda^{\prime} \in Z_{n, D}$ with $d(\Lambda) \neq d\left(\Lambda^{\prime}\right)$, then $\left(\mathbf{P}_{ \pm}\right)_{\Lambda, \Lambda^{\prime}}=0$ and $\boldsymbol{\Lambda}_{\Lambda, \Lambda^{\prime}}=0$.

Proof. We consider the matrix equation (4.2.8). It is clear that the right hand side of (4.2.8) coincides with the matrix $\boldsymbol{\Omega}$ defined in 4.3. On the other hand, $\mathbf{P}_{ \pm}=K_{ \pm}(\mathbf{t}), \Lambda(\mathbf{t})=$ $\boldsymbol{\Lambda}$ satisfies the condition (a) and (b) in (i). Thus $K_{ \pm}(\mathbf{t})$ and $\Lambda(\mathbf{t})$ satisfies the equation (4.4.1). We now consider (4.4.1) as an equation of matrices, where $\boldsymbol{\Omega}$ is known, and $\mathbf{P}_{ \pm}, \boldsymbol{\Lambda}$ are unknown matrices. By a standard argument from the linear algebra, we know that $\mathbf{P}_{ \pm}$ and $\boldsymbol{\Lambda}$ are the unique solution of (4.4.1) and that there exists an algorithm of computing $\mathbf{P}_{ \pm}$and $\boldsymbol{\Lambda}$. Thus (i) holds.

Next we show (ii). The proof below is a natural generalization of the argument in the proof of [L4, Thm 24.4]. We write $\left(\mathbf{P}_{ \pm}\right)_{\Lambda, \Lambda^{\prime}}=p_{\Lambda, \Lambda^{\prime}}^{ \pm}$and $\boldsymbol{\Lambda}_{\Lambda, \Lambda^{\prime}}=\lambda_{\Lambda, \Lambda^{\prime}}$. Let $\boldsymbol{\Lambda}_{\mathscr{C}}$ be the block corresponding to a similarity class $\mathscr{C}$. We consider the following statement. 
$\left(A_{\mathscr{C}}\right)$ Any element $\lambda_{\Lambda, \Lambda^{\prime}}$ in $\boldsymbol{\Lambda}_{\mathscr{C}}$ satisfies (ii).

$\left(B_{\mathscr{C}}^{ \pm}\right) p_{\Lambda, \Lambda^{\prime}}^{ \pm}$satisfies (ii) for any $\Lambda^{\prime} \in \mathscr{C}$ and for any $\Lambda \in Z_{n, D}$.

We show, by induction, that $\left(A_{\mathscr{C}}\right)$ and $\left(B_{\mathscr{C}}^{ \pm}\right)$holds for any class $\mathscr{C}$.

Take $\Lambda, \Lambda^{\prime} \in Z_{n, D}$, such that $d(\Lambda) \neq d\left(\Lambda^{\prime}\right)$. Then (4.4.1) can be written as

$$
\sum_{\Lambda \succeq \Lambda_{1}} \sum_{\Lambda^{\prime} \succeq \Lambda_{2}} p_{\Lambda, \Lambda_{1}}^{-} \lambda_{\Lambda_{1}, \Lambda_{2}} p_{\Lambda^{\prime}, \Lambda_{2}}^{+}=\omega_{\Lambda, \Lambda^{\prime}}=0
$$

First we show that

(4.4.3) Assume that $\left(A_{\mathscr{C}^{\prime}}\right)$ holds for $\mathscr{C}^{\prime} \prec \mathscr{C}$, and $\left(B_{\mathscr{C}^{\prime}}^{ \pm}\right)$holds for $\mathscr{C}^{\prime} \prec \mathscr{C}$. Then $\left(A_{\mathscr{C}}\right)$ holds. In particular, $\left(A_{\mathscr{C}}\right)$ holds if $\mathscr{C}$ is the minimum class in $Z_{n, D}$.

Take $\Lambda, \Lambda^{\prime} \in \mathscr{C}$. We note that

$$
\sum_{\substack{\Lambda \succeq \Lambda_{1} \\ \Lambda^{\prime} \succeq \Lambda_{2}}} \sum_{\substack{\Lambda \ngtr \Lambda_{1} \\ \text { or } \\ \Lambda^{\prime} \nsim \Lambda_{2}}} p_{\Lambda, \Lambda_{1}}^{-} \lambda_{\Lambda_{1}, \Lambda_{2}} p_{\Lambda^{\prime}, \Lambda_{2}}^{+}=0
$$

under the assumption of (4.4.3). In fact, suppose that the left hand side of (4.4.4) is not zero. Then there exists $\Lambda_{1}, \Lambda_{2}$ such that $p_{\Lambda, \Lambda_{1}}^{-} \neq 0, \lambda_{\Lambda_{1}, \Lambda_{2}} \neq 0, p_{\Lambda^{\prime}, \Lambda_{2}}^{+} \neq 0$. If $\Lambda \nsim \Lambda_{1}$, we have $d(\Lambda)=d\left(\Lambda_{1}\right)$ by (4.4.3). Since $\lambda_{\Lambda_{1}, \Lambda_{2}} \neq 0$, then $\Lambda_{1} \sim \Lambda_{2}$. Thus by (4.4.3), we have $d\left(\Lambda_{1}\right)=d\left(\Lambda_{2}\right)$ and $d\left(\Lambda_{2}\right)=d\left(\Lambda^{\prime}\right)$. This implies that $d(\Lambda)=d\left(\Lambda^{\prime}\right)$, which contradicts to our assumption. The case where $\Lambda^{\prime} \nsim \Lambda_{2}$ is proved similarly. Hence (4.4.4) holds.

Substituting the equation (4.4.4) into (4.4.2), by noting that the diagonal block of $\mathbf{P}_{ \pm}$ corresponding to $\mathscr{C}$ is the identity matrix, we have

$$
\omega_{\Lambda, \Lambda^{\prime}}=\lambda_{\Lambda, \Lambda^{\prime}}=0
$$

Hence $\left(A_{\mathscr{C}}\right)$ holds.

Next we show that

(4.4.6) Assume that $\left(A_{\mathscr{C}^{\prime}}\right)$ holds for any $\mathscr{C}^{\prime} \preceq \mathscr{C}$, and $\left(B_{\mathscr{C}^{\prime}}^{ \pm}\right)$holds for any $\mathscr{C}^{\prime} \prec \mathscr{C}$. Then $\left(B_{\mathscr{C}}^{ \pm}\right)$holds.

Take $\Lambda \in Z_{n, D}$ and $\Lambda^{\prime} \in \mathscr{C}$. Under the assumption of (4.4.6), the following equation can be proved in a similar way as in (4.4.4).

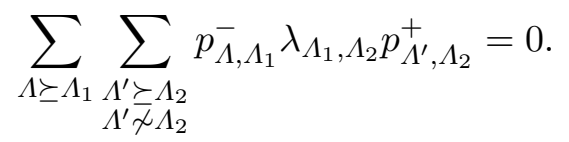

By substituting (4.4.7) into (4.4.2), we have

$$
\sum_{\substack { \Lambda \succeq \Lambda_{1} \\
\begin{subarray}{c}{\Lambda^{\prime} \succeq \Lambda_{2} \\
\Lambda^{\prime} \sim \Lambda_{2}{ \Lambda \succeq \Lambda _ { 1 } \\
\begin{subarray} { c } { \Lambda ^ { \prime } \succeq \Lambda _ { 2 } \\
\Lambda ^ { \prime } \sim \Lambda _ { 2 } } }\end{subarray}} p_{\Lambda, \Lambda_{1}}^{-} \lambda_{\Lambda_{1}, \Lambda_{2}} p_{\Lambda^{\prime}, \Lambda_{2}}^{+}=0 .
$$


Since the diagonal block of $\mathbf{P}_{+}$corresponding to $\mathscr{C}$ is the identity matrix, and since $\left(A_{\mathscr{C}}\right)$ holds, (4.4.8) can be written as

$$
\sum_{\substack{\Lambda_{1} \in \mathscr{C} \\ d\left(\Lambda_{1}\right)=d\left(\Lambda^{\prime}\right)}} p_{\Lambda, \Lambda_{1}}^{-} \lambda_{\Lambda_{1}, \Lambda^{\prime}}=0
$$

This holds for any $\Lambda \in Z_{n, D}$ and $\Lambda^{\prime} \in \mathscr{C}$. For a fixed $\Lambda$, we consider (4.4.9) as a system of linear equations with unknown variables $p_{\Lambda, \Lambda_{1}}^{-}$. Since $\boldsymbol{\Lambda}_{\mathscr{C}}$ is a non-singular matrix, the submatrix $\left(\lambda_{\Lambda_{1}, \Lambda^{\prime}}\right)_{d\left(\Lambda_{1}\right)=d\left(\Lambda^{\prime}\right)}$ is also non-singular (it gives a block decomposition of $\boldsymbol{\Lambda}_{\mathscr{C}}$ under a suitable permutation of indices). It follows that $p_{\Lambda, \Lambda_{1}}^{-}=0$ if $d(\Lambda) \neq d\left(\Lambda_{1}\right)$, and $\left(B_{\mathscr{C}}^{-}\right)$holds. If we take $\Lambda \in \mathscr{C}$ and $\Lambda^{\prime} \in Z_{n, D}$, by a similar argument as above, one can show that $\left(B_{\mathscr{C}}^{+}\right)$holds. Hence (4.4.6) is proved.

Now $\left(A_{\mathscr{C}}\right)$ and $\left(B_{\mathscr{C}}^{ \pm}\right)$follows from (4.4.3) and (4.4.6). The assertion (ii) is proved.

4.5. In the one-parameter case, we obtain a more precise result than Theorem 4.4. Hereafter, we consider the one-parameter case.

For $W=W_{n, r}$, we denote by $P_{W}(t)$ the Poincaré polynomial of $W$. Explicitly, it is given as

$$
P_{W}(t)=\prod_{i=1}^{n} \frac{t^{i r}-1}{t-1}
$$

Let $\mathbf{V}$ be the $n$-dimensional vector space over $\mathbf{C}$ such that $\mathbf{V}$ is the reflection representation of $W$. For any character $f$ of $W$, put

$$
R(f)=(t-1)^{n} P_{W}(t)|W|^{-1} \sum_{w \in W} \frac{\operatorname{det}_{\mathbf{V}}(w) f(w)}{\operatorname{det}_{\mathbf{V}}\left(t \cdot \operatorname{id}_{\mathbf{V}}-w\right)}
$$

If we denote by $R_{W}=\bigoplus_{i} R_{i}$ the coinvariant algebra of $W$, we have $P_{W}(t)=\sum_{i \geq 0}\left(\operatorname{dim} R_{i}\right) t^{i}$, and $R(f)=\sum_{i}\left\langle R_{i}, f\right\rangle t^{i}$ (the graded multiplicity of $f$ in $R_{W}$ ). Hence $R(f) \in \mathbf{Z}[t]$. Let $N^{*}$ be the number of reflections in $W$. Then $N^{*}$ is the highest degree of $R_{W}$, and $R_{N^{*}} \simeq \overline{\operatorname{det}}_{\mathbf{V}}$ as $W$-modules. Explicitly, we have

$$
N^{*}=\sum_{i=1}^{n}(r i-1)=r n(n+1) / 2-n .
$$

For any $\boldsymbol{\lambda}, \boldsymbol{\mu} \in \mathscr{P}_{n, r}$, we define $\widetilde{\omega}_{\boldsymbol{\lambda}, \boldsymbol{\mu}}^{\prime}(t) \in \mathbf{Q}(t)$ by

$$
\widetilde{\omega}_{\boldsymbol{\lambda}, \boldsymbol{\mu}}^{\prime}(t)=\frac{t^{n}}{|W|} \sum_{w \in W} \frac{\chi^{\boldsymbol{\lambda}}(w) \bar{\chi}^{\boldsymbol{\mu}}(w)}{\operatorname{det}_{\mathbf{V}}\left(t \cdot \operatorname{id}_{\mathbf{V}}-w\right)}
$$

We define $\mathbb{G}_{W}(t) \in \mathbf{Z}[t]$ by $\mathbb{G}_{W}(t)=(t-1)^{n} t^{N^{*}} P_{W}(t)$. ( $\mathbb{G}_{W}(t)$ is regarded as a formal analogue of the order of the finite classical group $G\left(\mathbf{F}_{q}\right)$, where the role of the Weyl group 
is replaced by the complex reflection group $W_{n, r}($ see $\left.[\mathrm{BMM}]).\right) \widetilde{\omega}_{\boldsymbol{\lambda}, \boldsymbol{\mu}}^{\prime}(t)$ can be written as

$$
\widetilde{\omega}_{\boldsymbol{\lambda}, \boldsymbol{\mu}}^{\prime}(t)=\mathbb{G}_{W}(t)^{-1} t^{N^{*}+n} R\left(x^{\boldsymbol{\lambda}} \otimes \bar{\chi}^{\boldsymbol{\mu}} \otimes \overline{\operatorname{det}}_{\mathbf{V}}\right) .
$$

For $\Lambda, \Lambda^{\prime} \in Z_{n, D}$, we define $\widetilde{\omega}_{\Lambda, \Lambda^{\prime}}(t)$ as follows. Put $W=W_{n, r}$.

$$
\widetilde{\omega}_{\Lambda, \Lambda^{\prime}}(t)= \begin{cases}\mathbb{G}_{W}(t) \widetilde{\omega}_{\boldsymbol{\lambda}, \boldsymbol{\mu}}^{\prime}(t) & \text { if } \Lambda=\Lambda(\boldsymbol{\lambda}), \Lambda^{\prime}=\Lambda(\boldsymbol{\mu}) \text { with } \boldsymbol{\lambda}, \boldsymbol{\mu} \in \mathscr{P}_{n^{\prime}, r}, \\ 0 & \text { if } d(\Lambda) \neq d\left(\Lambda^{\prime}\right) .\end{cases}
$$

Note that in the first case, $\omega_{\boldsymbol{\lambda}, \boldsymbol{\mu}}^{\prime}(t)$ is defined with respect to $W^{\prime}=W_{n^{\prime}, r}$. Since $\mathbb{G}_{W}(t)$ is divisible by $\mathbb{G}_{W^{\prime}}(t)$, we see that $\widetilde{\omega}_{\Lambda, \Lambda^{\prime}}(t) \in \mathbf{Z}[t]$.

We define a matrix $\widetilde{\boldsymbol{\Omega}}$ by $\widetilde{\boldsymbol{\Omega}}=\left(\widetilde{\omega}_{\Lambda, \Lambda^{\prime}}(t)\right)$. Note that $\widetilde{\boldsymbol{\Omega}}$ can be computed explicitly once we know the character table of $W_{n^{\prime}, r}$ for various $n^{\prime} \leq n$.

Let $\widetilde{K}_{\Lambda, \Lambda^{\prime}}^{ \pm}(t)$ be the modified Kostka functions defined in (3.10.3). Computing $K_{\Lambda, \Lambda^{\prime}}^{ \pm}(t)$ is equivalent to computing $\widetilde{K}_{\Lambda, \Lambda^{\prime}}^{ \pm}(t)$. We define a matrix $\widetilde{K}_{ \pm}(t)$ by $\widetilde{K}_{ \pm}(t)=\left(\widetilde{K}_{\Lambda, \Lambda^{\prime}}^{ \pm}(t)\right)$. By a similar argument as in the proof of Theorem 4.4, we have the following result, which is a generalization of [S2, Thm. 5.4].

Theorem 4.6. (i) The matrix $\widetilde{K}_{ \pm}(t)$ is determined as the unique solution of the following equation satisfying the properties (a) and (b).

$$
\widetilde{\mathbf{P}}_{-} \widetilde{\boldsymbol{\Lambda}}^{t} \widetilde{\mathbf{P}}_{+}=\widetilde{\boldsymbol{\Omega}} \text {. }
$$

(a) $\widetilde{\mathbf{P}}_{ \pm}$is a lower triangular block matrix, where the diagonal block is the scalar matrix $t^{a(\Lambda)} I_{\mathscr{C}}$ for the similarity class $\mathscr{C}$ containing $\Lambda$.

(b) $\widetilde{\boldsymbol{\Lambda}}$ is a diagonal block matrix.

Then $\widetilde{K}_{ \pm}=\widetilde{\mathbf{P}}_{ \pm}$.

(ii) If $\Lambda, \Lambda^{\prime} \in Z_{n, D}$ with $d(\Lambda) \neq d\left(\Lambda^{\prime}\right)$, then $\left(\widetilde{\mathbf{P}}_{ \pm}\right)_{\Lambda, \Lambda^{\prime}}=0$ and $\widetilde{\boldsymbol{\Lambda}}_{\Lambda, \Lambda^{\prime}}=0$.

Proof. We consider the formula (4.2.8), specialized to $\mathbf{t}=\mathbf{t}_{0}$. We write $K_{ \pm}\left(\mathbf{t}_{0}\right), \Lambda\left(\mathbf{t}_{0}\right)$, $Z\left(\mathbf{t}_{0}\right)$ as $K_{ \pm}(t), \Lambda(t), Z(t)$, and write $X(\mathbf{0})=X(0)$. We will translate this formula to the formula with respect to modified Kostka functions. It follows from the definition of $\widetilde{K}_{\Lambda, \Lambda^{\prime}}^{ \pm}(t)$, one can write as $\widetilde{K}_{ \pm}(t)=K_{ \pm}\left(t^{-1}\right) S$, where $S$ is the diagonal matrix whose $\Lambda \Lambda$-entry is $t^{a(\Lambda)}$. Then (4.2.8) is rewritten as

$$
\widetilde{K}_{-}(t) \Lambda^{\prime}(t){ }^{t} \widetilde{K}_{+}(t)=\bar{X}(0)^{-1} H Z\left(t^{-1}\right)^{-1} H^{t} X(0)^{-1},
$$

where $\Lambda^{\prime}(t)=S^{-1} \Lambda\left(t^{-1}\right) S^{-1}$. Let $M=\left(M_{\Lambda, \Lambda^{\prime}}\right)$ be the right hand side of (4.6.2). As in the proof of Theorem 4.4, we see that $M_{\Lambda, \Lambda^{\prime}}=0$ if $d(\Lambda) \neq d\left(\Lambda^{\prime}\right)$.

Here we note the following relation. If $w_{\boldsymbol{\lambda}}$ is an element in $W_{n^{\prime}, r}$ with type $\boldsymbol{\lambda} \in \mathscr{P}_{n^{\prime}, r}$, we have

$$
\operatorname{det}_{\mathbf{V}}\left(t \cdot \operatorname{id}_{\mathbf{V}}-w_{\boldsymbol{\lambda}}\right)=\prod_{k=1}^{r} \prod_{j=1}^{l\left(\lambda^{(k)}\right)}\left(t^{\lambda_{j}^{(k)}}-\zeta^{k-1}\right)
$$


By comparing this with the definition of $z_{\boldsymbol{\lambda}}(t)$ in (2.7.1) and (2.7.2), we have

$$
z_{\boldsymbol{\lambda}}\left(t^{-1}\right)=\frac{z_{\boldsymbol{\lambda}} t^{n^{\prime}}}{\operatorname{det}_{\mathbf{V}}\left(t \cdot \operatorname{id}_{\mathbf{V}}-w_{\boldsymbol{\lambda}}\right)}
$$

Assume that $\Lambda=\Lambda(\boldsymbol{\lambda}), \Lambda^{\prime}=\Lambda(\boldsymbol{\mu})$ with $\boldsymbol{\lambda}, \boldsymbol{\mu} \in \mathscr{P}_{n^{\prime}, r}$. Note that $Z(t)$ is a diagonal matrix with $\Lambda \Lambda$-entry $z_{\Lambda}(t)^{-1}$. Substituting (4.6.4) into $Z\left(t^{-1}\right)^{-1}$, we can compute $M_{\Lambda, \Lambda^{\prime}}$. By (4.6.2), we have

$$
M_{\Lambda, \Lambda^{\prime}}=t^{n^{\prime}}\left|W^{\prime}\right|^{-1} \sum_{w \in W^{\prime}} \frac{\chi^{\boldsymbol{\lambda}}(w) \bar{\chi}^{\boldsymbol{\mu}}(w)}{\operatorname{det}_{\mathbf{V}}\left(t \cdot \operatorname{id}_{\mathbf{V}}-w\right)}=\widetilde{\omega}_{\boldsymbol{\lambda}, \boldsymbol{\mu}}^{\prime}(t) .
$$

Hence if we put $\widetilde{\Lambda}(t)=\mathbb{G}_{W}(t) \Lambda^{\prime}(t)$, by (4.6.2), we obtain the required formula

$$
\widetilde{K}_{-}(t) \widetilde{\Lambda}(t)^{t} \widetilde{K}_{+}(t)=\widetilde{\Omega} .
$$

Since $\widetilde{K}_{ \pm}(t), \widetilde{\Lambda}(t)$ satisfy the condition (a), (b), the first assertion is proved. The assertion (ii) is proved in a similar way as in the proof of Theorem 4.4.

\section{Generalized Green functions of Symplectic groups}

5.1. Let $G$ be a connected reductive group over $\mathbf{k}$, where $\mathbf{k}$ is an algebraic closure of a finite field $\mathbf{F}_{q}$ of $q$-elements, and $F: G \rightarrow G$ the Frobenius map corresponding to $\mathbf{F}_{q}$, so that $G^{F}=G\left(\mathbf{F}_{q}\right)$ is a finite reductive group. We denote by $G_{\text {uni }}$ the set of unipotent elements in $G$, and $G_{\text {uni }}^{F}$ the set of unipotent elements in $G^{F}$. Let $\mathscr{N}_{G}$ be the set of pairs $(C, \mathscr{E})$, where $C$ is a unipotent class in $G$ and $\mathscr{E}$ is a $G$-equivariant simple local system on $C$. We denote by $\mathscr{N}_{G}^{0}$ the subset of $\mathscr{N}_{G}$ consisting of $(C, \mathscr{E})$ such that $\mathscr{E}$ is a cuspidal local system on $C([\mathrm{~L} 2])$. Let $\mathscr{S}_{G}$ be the set of triples $\left(L, C_{1}, \mathscr{E}_{1}\right)$, up to $G$-conjugate, where $L$ is a Levi subgroup of a parabolic subgroup $P$ of $G$, and $\left(C_{1}, \mathscr{E}_{1}\right) \in \mathscr{N}_{L}^{0}$. Take an $F$-stable $\left(L, C_{1}, \mathscr{E}_{1}\right) \in \mathscr{S}_{G}$, namely, the triple such that $F(L)=L, F\left(C_{1}\right)=C_{1}$ and $F^{*} \mathscr{E} 1 \simeq \mathscr{E}_{1}$. We fix an isomorphism $\varphi_{1}: F^{*} \mathscr{E}_{1} \underset{\rightarrow}{\rightarrow} \mathscr{E}_{1}$. In [L3, 8.3], Lusztig constructed the semisimple perverse sheaf $K$ on $G$, associated to $\left(L, C_{1}, \mathscr{E}_{1}\right)$, and the isomorphism $\varphi: F^{*} K \stackrel{\sim}{\rightarrow} K$ induced from $\varphi_{1}$. He defined the generalized Green function $Q_{L, C_{1}, \mathscr{E}_{1}, \varphi_{1}}^{G}$ as the restriction to $G_{\text {uni }}^{F}$ of the characteristic function $\chi_{K, \varphi}$ on $G^{F}$, which is a $G^{F}$-invariant function on $G_{\text {uni }}^{F}$ with values in $\overline{\mathbf{Q}}_{l}$.

In the case where $G=G L_{n}$, an $F$-stable maximal torus is written as $T_{w}$, twisted from the split maximal torus $T_{0}$ by $w \in S_{n}$. The $G^{F}$-conjugacy classes of $G_{\text {uni }}^{F}$ are parametrized by $\mathscr{P}_{n}$, we denote by $u_{\nu} \in G_{\text {uni }}^{F}$ a representative of the class corresponding to $\nu \in \mathscr{P}_{n}$. As the special case of $Q_{L, C_{1}, \mathscr{E}_{1}, \varphi_{1}}^{G}$, we have the Green function $Q_{T_{w}}^{G}$ on $G_{\text {uni }}^{F}$, which satisfies the relation

$$
Q_{T_{w_{\mu}}}^{G}\left(u_{\nu}\right)=Q_{\mu, \nu}(q)=\sum_{\lambda \in \mathscr{P}_{n}} \chi^{\lambda}\left(w_{\mu}\right) \widetilde{K}_{\lambda \nu}(q)
$$


where $\chi^{\lambda}$ is the irreducible character of $S_{n}$ corresponding to $\lambda \in \mathscr{P}_{n}$, and $Q_{\mu, \nu}(t)$ is as in 4.2 .

In view of the discussion in 4.2, (5.1.1) gives a combinatorial description of Green functions $Q_{T_{w}}^{G}$ in terms of Green polynomials $Q_{\mu, \nu}(t)$, or equivalently, of Kostka polynomials $K_{\lambda \nu}(t)$. In this section, we show, in the case of symplectic groups, that generalized Green functions can be described in a combinatorial way in terms of our generalized Green functions defined in 4.2, or equivalently, of Kostka functions $K_{\Lambda, \Lambda^{\prime}}(t)$.

5.2. From now on, assume that $G$ is the symplectic group $S p_{2 n}$ (with any characteristic). In this case, all the pairs $(C, \mathscr{E}) \in \mathscr{N}_{G}$ are $F$-stable. It is known that the set $\mathscr{N}_{G}$ is described in terms of 2 -symbols ([L2] for $\operatorname{ch} \mathbf{k} \neq 2$, [LS] for $\operatorname{ch} \mathbf{k}=2$ ). We consider the 2-symbols $Z_{n, D}^{e, \mathbf{s}}$ as follows. Put $e=2, \mathbf{s}=(0,1)$ in the case where $\operatorname{ch} \mathbf{k} \neq 2$, and put $e=4, \mathbf{s}=(0,2)$ in the case where $\operatorname{ch} \mathbf{k}=2$. In either case assume that $\alpha=1$, namely, $m^{\bullet}=(m+1, m)$, and let $D$ be the set of defects $\mathbf{d}=(d, 0)$ or $(0, d)$ such that $d$ is odd. Then $Z_{n, D}^{e, \mathbf{s}}$ coincides with the set $X_{n}^{1,1}\left(\right.$ resp. $\left.X_{n}^{2,2}\right)$ if $\operatorname{ch} \mathbf{k} \neq 2($ resp. $\operatorname{ch} \mathbf{k}=2)$ in the notation in [LS]. Hereafter we write $Z_{n, D}^{e, \mathbf{s}}$ as $Z_{n, D}$. It is proved in [L2], [LS] that $\mathscr{N}_{G}$ is in bijection with $Z_{n, D}$ such that the unipotent classes in $G_{\text {uni }}$ correspond to the similarity classes in $Z_{n, D}$. Moreover, the set $D$ is in bijection with the set $\mathscr{S}_{G}$. For $\left(L, C_{1}, \mathscr{E}_{1}\right) \in \mathscr{S}_{G}$ with $L \simeq\left(G L_{1}\right)^{n^{\prime}} \times S p_{2 n-2 n^{\prime}}, N_{G}(L) / L \simeq W_{n^{\prime}, 2}$, there exists a natural bijection $Z_{n, \mathbf{d}} \underset{\rightarrow}{\rightarrow} W_{n^{\prime}, 2}^{\wedge}$, which we denote by $\Lambda \mapsto \chi^{\Lambda}$, such that the following generalized Springer correspondence holds ([L2, Thm. 12.3], [LS, Thm. 2.4]).

$$
\coprod_{\left(L, C_{1}, \mathscr{E}_{1}\right) \in \mathscr{S}_{G}}\left(N_{G}(L) / L\right)^{\wedge} \simeq \coprod_{\mathbf{d} \in D} Z_{n, \mathbf{d}}=Z_{n, D}
$$

5.3. Let $\left(L, C_{1}, \mathscr{E}_{1}\right) \in \mathscr{S}_{G}$ be corresponding to $\mathbf{d} \in D$. Put $\mathscr{W}_{L}=N_{G}(L) / L$ and let $Z(L)$ be the center of $L$. Then $K$ can be decomposed into simple components

$$
K \simeq \bigoplus_{\Lambda \in Z_{n, \mathbf{d}}} V_{\Lambda} \otimes A_{\Lambda}
$$

where $V_{\Lambda}$ is an irreducible $\mathscr{W}_{L}$-module with character $\chi^{\Lambda}$, and $A_{\Lambda}$ is a simple perverse sheaf on $G$. It is known that $\left.A_{\Lambda}\right|_{G_{\text {uni }}} \simeq \operatorname{IC}\left((\bar{C}, \mathscr{E})\left[a_{\Lambda}\right]\right.$, where $a_{\Lambda}=-\operatorname{dim} C-\operatorname{dim} Z(L)$, under the correspondence $\Lambda \leftrightarrow(C, \mathscr{E})$. By (5.3.1), $\varphi: F^{*} K \stackrel{\sim}{\rightarrow} K$ induces a canonical isomorphism $\varphi_{\Lambda}: F^{*} A_{\Lambda} \underset{\rightarrow}{\rightarrow} A_{\Lambda}$ for $\Lambda \in Z_{n, \mathbf{d}}$ (which corresponds to $\phi_{A_{i}}$ in [L4, 24.2]). We now define a function $X_{\Lambda}$ on $\bar{C}^{F}$ by

$$
X_{\Lambda}(g)=\sum_{a}(-1)^{a+a_{\Lambda}} \operatorname{Tr}\left(\varphi_{\Lambda}, \mathscr{H}_{g}^{a} A_{\Lambda}\right) q^{-\left(a_{\Lambda}+r_{\Lambda}\right) / 2},
$$

for $g \in \bar{C}^{F}$, where $r_{\Lambda}=\operatorname{dimsupp} A_{\Lambda} .\left(X_{\Lambda}\right.$ corresponds to $X_{i}$ in $\left.[\mathrm{L} 4,24.2]\right)$. Note that $a_{\Lambda}+r_{\Lambda}=(\operatorname{dim} G-\operatorname{dim} C)-\left(\operatorname{dim} L-\operatorname{dim} C_{1}\right)$.

There exists an $F$-stable parabolic subgroup $P_{0}$ and an $F$-stable Levi subgroup $L_{0}$ of $P_{0}$, and $F$ acts trivially on $\mathscr{W}_{L}=N_{G}\left(L_{0}\right) / L_{0} \simeq W_{n^{\prime}, 2}$. Moreover any $F$-stable Levi subgroup $L$ can be obtained as $L_{w}$, by twisting $L_{0}$ by $w \in \mathscr{W}_{L}$. For a pair $(C, \mathscr{E}) \in \mathscr{N}_{G}$ 
corresponding to $\Lambda \in Z_{n, \mathbf{d}}$, the generalized Green function $Q_{L_{w}, C_{1}, \mathscr{E}_{1}, \varphi_{1}}^{G}$ can be written as

$$
Q_{L_{w}, C_{1}, \mathscr{E}_{1}, \varphi_{1}}^{G}=\sum_{\Lambda \in Z_{n, \mathbf{d}}} \chi^{\Lambda}(w) X_{\Lambda} q^{\left(a_{\Lambda}+r_{\Lambda}\right) / 2}
$$

Thus the determination of $Q_{L, C_{1}, \mathscr{E}_{1}, \varphi_{1}}^{G}$ is reduced to the determination of various functions $X_{\Lambda}$ on $G_{\text {uni }}^{F}$ for $\Lambda \in Z_{n, \mathbf{d}}$.

5.4. Since $\left.\mathscr{H}^{a_{\Lambda}} A_{\Lambda}\right|_{G_{\text {uni }}} \simeq \mathscr{E}, q^{-\left(a_{\Lambda}+r_{\Lambda}\right)} \varphi_{\Lambda}: F^{*} \mathscr{H}^{a_{\Lambda}} A_{\Lambda} \rightarrow \mathscr{H}^{a_{\Lambda}} A_{\Lambda}$ induces an isomorphism $F^{*} \mathscr{E} \simeq \mathscr{E}$, which we denote by $\psi_{\Lambda}$. It is known (under a suitable choice of $\left.\varphi_{1}\right)$ that $\psi_{\Lambda}$ induces an automorphism of finite order $\mathscr{E}_{g} \rightarrow \mathscr{E}_{g}$ for any $g \in C^{F}$. We denote by $Y_{\Lambda}$ the characteristic function $\chi_{\mathscr{E}, \psi_{\Lambda}}$ on $C^{F}$, extended to the function on $G_{\text {uni }}^{F}$, namely

$$
Y_{\Lambda}(g)= \begin{cases}\operatorname{Tr}\left(\psi_{\Lambda}, \mathscr{E}_{g}\right) & \text { if } g \in C^{F} \\ 0 & \text { if } g \notin C^{F}\end{cases}
$$

Then $\left\{Y_{\Lambda} \mid \Lambda \in Z_{n, D}\right\}$ gives rise to a basis of the $\overline{\mathbf{Q}}_{l}$-space of $G^{F}$-invariant functions of $G_{\text {uni }}^{F}$. We express $X_{\Lambda}$ as $X_{\Lambda}=\sum_{\Lambda^{\prime} \in Z_{n, D}} p_{\Lambda, \Lambda^{\prime}} Y_{\Lambda^{\prime}}$ with $p_{\Lambda, \Lambda^{\prime}} \in \overline{\mathbf{Q}}_{l}$, and consider the matrix $P=\left(p_{\Lambda, \Lambda^{\prime}}\right)$ indexed by $\Lambda, \Lambda^{\prime} \in Z_{n, D}$. We define a total order $\preceq$ on $Z_{n, D}$ such that each similarity class gives an interval, and that $\Lambda^{\prime} \prec \Lambda$ if $C^{\prime} \subset \bar{C}$, where $C$ (resp. $C^{\prime}$ ) corresponds to the similarity class containing $\Lambda$ (resp. $\Lambda^{\prime}$ ). (Note that in our case $a(\Lambda)$ coincides with $\left(\operatorname{dim} G_{\text {uni }}-\operatorname{dim} C\right) / 2$ by [L5, 4.4], this agrees with the choice of $\preceq$ in 3.5.) The following theorem was proved by Lusztig. In the following, we consider the matrices with respect to the total order $\preceq$, and regard them as block matrices according to the partition of $Z_{n, D}$ by similarity classes.

Theorem 5.5 (Lusztig [L4, Thm. 24.4]). The matrix $P$ is obtained as the unique solution of the equation

$$
P \Lambda^{t} P=\Omega \text {. }
$$

Here $P$ is a lower triangular block matrix, where the diagonal block is the identity matrix, and $\Lambda$ is a diagonal block matrix. Moreover, $\Omega=\left(\omega_{\Lambda, \Lambda^{\prime}}\right)$ is defined as

$$
\omega_{\Lambda, \Lambda^{\prime}}=\frac{q^{-\operatorname{dim} G} q^{-\left(a_{\Lambda}+a_{\Lambda^{\prime}}\right) / 2}\left|G^{F}\right|}{\left|\mathscr{W}_{L}\right|} \sum_{w \in \mathscr{W}_{L}} \frac{\chi^{\Lambda}(w) \chi^{\Lambda^{\prime}}(w)}{\left|Z\left(L_{w}\right)^{F}\right|}
$$

if $d(\Lambda)=d\left(\Lambda^{\prime}\right)$, and $\omega_{\Lambda, \Lambda^{\prime}}=0$ if $d(\Lambda) \neq d\left(\Lambda^{\prime}\right)$.

5.6. Theorem 5.5 shows that there is an algorithm of computing the matrix $P$. Thus the determination of $Q_{L, C_{1}, \mathscr{E}_{1}, \varphi_{1}}^{G}$ is reduced to the determination of the functions $Y_{\Lambda}$. Note that the definition of $Y_{\Lambda}$ depends on the choice of $\varphi_{1}$. Even if $\Lambda \sim \Lambda^{\prime}$ belong to the same unipotent class $C, Y_{\Lambda}$ and $Y_{\Lambda^{\prime}}$ have no relations if $d(\Lambda) \neq d\left(\Lambda^{\prime}\right)$. However, we can show that there exists a canonical choice of $Y_{\Lambda}$. In order to describe $Y_{\Lambda}$, we need to choose a good representative for each class $C^{F}$. In [S1], it was shown, in the case where $\operatorname{ch} \mathbf{k} \neq 2$, that there exists good representatives in $C^{F}$, called split elements, which behave well with respect to the computation of Green functions, namely the case where $L$ is a maximal torus $T, C_{1}$ is the identity class $\{1\}$, and $\mathscr{E}_{1}$ is the constant sheaf $\overline{\mathbf{Q}}_{l}$. By using 
split elements, $Y_{\Lambda}$ can be computed for $\Lambda \in Z_{n, \mathbf{d}} \bullet$. In [S5], it was shown that those split elements behave well also for any triple $\left(L, C_{1}, \mathscr{E}_{1}\right)$. Also the case of $\mathrm{ch} \mathbf{k}=2$ was discussed there.

In the case of symplectic groups, if we fix $u \in C^{F}$, and put $A(u)=Z_{G}(u) / Z_{G}^{0}(u)$, then $A(u)$ is an abelian group on which $F$ acts trivially. The $G^{F}$-classes of $C^{F}$ are parametrized by $A(u)$. We denote by $C_{a}^{F}$ the $G^{F}$-class in $C^{F}$ corresponding to $a \in A(u)$. Also any $G$ equivariant simple local system $\mathscr{E}$ on $C$ is parametrized by $A(u)^{\wedge}$. We write $\mathscr{E} \rho$ the local system on $C$ corresponding to $\rho \in A(u)^{\wedge}$. We have the following result.

Theorem 5.7 ([S5, Cor. 4.4]). Assume that $G=S p_{2 n}$. There exists a good representative for $C^{F}$, called the split element, satisfying the following properties. For any $\left(L, C_{1}, \mathscr{E} 1\right) \in$ $\mathscr{S}_{G}^{F}$, take the split element $u_{1} \in C_{1}^{F}$, and define $\varphi_{1}: F^{*} \mathscr{E}_{1} \underset{\sim}{\rightarrow} \mathscr{E}_{1}$ so that the induced map $\left(\mathscr{E}_{1}\right)_{u_{1}} \rightarrow\left(\mathscr{E}_{1}\right)_{u_{1}}$ is the identity map. Then for any $(C, \mathscr{E}) \leftrightarrow \Lambda \in Z_{n, \mathbf{d}}, \psi_{\Lambda}: F^{*} \mathscr{E} \rightrightarrows \mathscr{E}$ satisfies the property that the induced map $\mathscr{E}_{u} \rightarrow \mathscr{E}_{u}$ is the identity map for the split element $u \in C^{F}$. In particular, if $\mathscr{E}=\mathscr{E}_{\rho}$ for $\rho \in A(u)^{\wedge}$, we have

$$
Y_{\Lambda}(g)= \begin{cases}\rho(a) & \text { if } g \in C_{a}^{F}, \\ 0 & \text { if } g \notin C^{F} .\end{cases}
$$

5.8. We consider the Kostka functions associated to the 2-symbols $Z_{n, D}$. As in 3.10 , we write them as $K_{\Lambda, \Lambda^{\prime}}(t), \widetilde{K}_{\Lambda, \Lambda^{\prime}}(t)$, etc. by ignoring the signature. The following result gives a combinatorial description of generalized Green functions.

Theorem 5.9. For any $\Lambda \in Z_{n, \mathbf{d}}$, the function $X_{\Lambda}$ can be written as

$$
X_{\Lambda}=\sum_{\Lambda^{\prime} \in Z_{n, D}} q^{-a(\Lambda)} \widetilde{K}_{\Lambda, \Lambda^{\prime}}(q) Y_{\Lambda^{\prime}}
$$

Proof. Here $\mathscr{W}_{L} \simeq W_{n^{\prime}, 2}$. If $w \in W_{n^{\prime}, 2}$ has type $(\nu, \sigma) \in \mathscr{P}_{n^{\prime}, 2}$ with $\nu: \nu_{1} \geq \cdots \geq \nu_{k}>0$, $\sigma: \sigma_{1} \geq \cdots \geq \sigma_{l}>0$, then we have

$$
\left|Z\left(L_{w}\right)^{F}\right|=\prod_{1 \leq i \leq k}\left(q^{\nu_{i}}-1\right) \prod_{1 \leq j \leq l}\left(q^{\sigma_{j}}+1\right)
$$

Hence $\left|Z\left(L_{w}\right)^{F}\right|=\operatorname{det}_{\mathbf{V}}\left(q \cdot \operatorname{id}_{\mathbf{V}}-w\right)$ by (4.6.3). Assume that $d(\Lambda)=d\left(\Lambda^{\prime}\right)$. Since $\left|G^{F}\right|=$ $\mathbb{G}_{W}(q)$ for $W=W_{n, 2}$, by comparing (5.5.1) with (4.5.4) for $W_{n^{\prime}, 2}$, we have

$$
\omega_{\Lambda, \Lambda^{\prime}}=q^{-\operatorname{dim} G-n^{\prime}} q^{-\left(a_{\Lambda}+a_{\Lambda^{\prime}}\right) / 2} \mathbb{G}_{W}(q) \widetilde{\omega}_{\Lambda, \Lambda^{\prime}}^{\prime}(q) .
$$

Since $a(\Lambda)=(\operatorname{dim} G-n-\operatorname{dim} C) / 2$ for any $\Lambda$ belonging to the class $C$, we have

$$
a_{\Lambda} / 2=-\left(\operatorname{dim} C+n^{\prime}\right) / 2=a(\Lambda)-\left(\operatorname{dim} G-n+n^{\prime}\right) / 2 .
$$

It follows that

$$
q^{a(\Lambda)+a\left(\Lambda^{\prime}\right)} \omega_{\Lambda, \Lambda^{\prime}}=q^{-n} \widetilde{\omega}_{\Lambda, \Lambda^{\prime}}(q) .
$$


On the other hand, we have $\omega_{\Lambda, \Lambda^{\prime}}=\widetilde{\omega}_{\Lambda, \Lambda^{\prime}}(q)=0$ if $d(\Lambda) \neq d\left(\Lambda^{\prime}\right)$.

Let $S$ be the diagonal matrix whose $\Lambda \Lambda$-entry equals to $q^{a(\Lambda)}$. It follows from (5.9.1) that we have an equation $(S P)\left(q^{n} \Lambda\right)^{t}(S P)=\widetilde{\boldsymbol{\Omega}}(q)$, which satisfies the same property as in (4.6.1), specialized to $t=q$. Thus by the uniqueness of the solution of (4.6.1), we have $S P=\widetilde{K}(q)$, namely, $p_{\Lambda, \Lambda^{\prime}}=q^{-a(\Lambda)} \widetilde{K}_{\Lambda, \Lambda^{\prime}}(q)$ for any $\Lambda, \Lambda^{\prime}$. The theorem is proved.

Remarks 5.10. (i) Theorem 5.9 implies that $\widetilde{K}_{\Lambda, \Lambda^{\prime}}(t)$, and so $K_{\Lambda, \Lambda^{\prime}}(t)$, do not depend on the choice of the total order $\preceq$ in 3.5 .

(ii) Theorem 5.9 also implies that $\widetilde{K}_{\Lambda, \Lambda^{\prime}}(t) \in \mathbf{Z}[t]$. In fact, $p_{\Lambda, \Lambda^{\prime}}=q^{-a(\Lambda)} \widetilde{K}_{\Lambda, \Lambda^{\prime}}(q)$ is regarded as a rational function on $q$, namely, there exists a rational function $p_{\Lambda, \Lambda^{\prime}}(t) \in \mathbf{Q}(t)$ such that $p_{\Lambda, \Lambda^{\prime}}(q)$ coincides with $p_{\Lambda, \Lambda^{\prime}}$. It is known by $[\mathrm{L} 4,(24.5 .2)]$ that $p_{\Lambda, \Lambda^{\prime}}\left(q^{s}\right)$ is an integer for any $s \in \mathbf{Z}_{\geq 0}$, which implies that $p_{\Lambda, \Lambda^{\prime}}(t)$ is a polynomial in $t$. Thus we have $\widetilde{K}_{\Lambda, \Lambda^{\prime}}(t) \in \mathbf{Z}[t]$. Note that in the case where $p$ is good, namely if $p \neq 2$, it was known by [L4, Thm. 24.8] that $p_{\Lambda, \Lambda^{\prime}}(q)$ is a polynomial in $q$. In the case where $p=2$, our result gives a weaker version of [L4, Thm. 24.8] (here the purity of the Frobenius eigenvalues are not assumed).

(iii) If $\operatorname{deg} \widetilde{K}_{\Lambda, \Lambda^{\prime}} \leq a\left(\Lambda^{\prime}\right)$, we have $K_{\Lambda, \Lambda^{\prime}}(t) \in \mathbf{Z}[t]$. It is likely that $K_{\Lambda, \Lambda^{\prime}}(t)$ is a polynomial with degree $a\left(\Lambda^{\prime}\right)-a(\Lambda)$.

5.11. Under the setting in 5.1, we consider the Green function $Q_{T, \varphi_{1}}^{G}$ on $G_{\text {uni }}^{F}$, which is a special case of the generalized Green functions $Q_{L, C_{1}, \mathscr{E}_{1}, \varphi_{1}}^{G}$, where $L=T$ is an $F$-stable maximal torus, $C_{1}=\{e\}, \mathscr{E} 1$ is the constant sheaf $\overline{\mathbf{Q}}_{l}$ on $C_{1}$, and $\varphi_{1}: F^{*} \overline{\mathbf{Q}}_{l} \stackrel{\sim}{\rightarrow} \overline{\mathbf{Q}}_{l}$ is the canonical isomorphism. For any integer $r \geq 1$, we can also consider $\varphi_{1}^{r}:\left(F^{r}\right)^{*} \overline{\mathbf{Q}}_{l} \underset{\sim}{\rightarrow} \overline{\mathbf{Q}}_{l}$, and the Green function $Q_{T, \varphi_{1}^{r}}^{G}$ on $G^{F^{r}}$. The following result was recently proved by $\mathrm{M}$. Geck $[\mathrm{G}]$ in a full generality.

Theorem 5.12 ([G]). Let $G$ be a connected reductive group defined over $\mathbf{F}_{q}$, and $u \in G^{F}$ a unipotent element. Assume that $r$ is a prime such that $r$ does not divide the order of $G^{F^{r}}$. Then

$$
Q_{T, \varphi_{1}}^{G}(u) \equiv Q_{T, \varphi_{1}^{r}}^{G}(u) \bmod r
$$

5.13. We consider a similar problem for the generalized Green function $Q_{L, C_{1}, \mathscr{E}_{1}, \varphi_{1}}^{G}$ on $G_{\text {uni }}^{F}$ for the case of symplectic groups. Fix an $F$-stable triple $\left(L, C_{1}, \mathscr{E}_{1}\right) \in \mathscr{S}_{G}$ as in 5.1. We choose a split element $u_{1} \in C_{1}^{F}$ and an isomorphism $\varphi_{1}: F^{*} \mathscr{E}_{1} \underset{\rightarrow}{\rightarrow} \mathscr{E}_{1}$ as in Theorem 5.7. Note that by [S5], if $u_{1}$ is split for $C_{1}^{F}$, then $u_{1}$ is also split for $C_{1}^{F^{r}}$ for any $r \geq 1$. We define $\varphi_{1}^{r}:\left(F^{r}\right)^{*} \mathscr{E} 1 \stackrel{\sim}{\rightarrow} \mathscr{E} 1$ by

$$
\varphi_{1}^{r}=\varphi \circ F^{*}\left(\varphi_{1}\right) \circ \cdots \circ\left(F^{*}\right)^{r-1}\left(\varphi_{1}\right):\left(F^{*}\right)^{r} \mathscr{E} 1 \stackrel{\sim}{\rightarrow} \cdots \stackrel{\sim}{\rightarrow}\left(F^{*}\right)^{2} \mathscr{E}_{1} \stackrel{\sim}{\rightarrow} F^{*} \mathscr{E}_{1} \stackrel{\sim}{\rightarrow} \mathscr{E} 1 .
$$

Then $\left(L, C_{1}, \mathscr{E}_{1}\right)$ is also $F^{r}$-stable, and one can consider the generalized Green function $Q_{L, C_{1}, \mathscr{E}_{1}, \varphi_{1}^{r}}^{G}$ on $G_{\text {uni }}^{F^{r}}$. Here note that for any $u \in G_{\text {uni }}^{F}, Q_{L, C_{1}, \mathscr{E}_{1}, \varphi_{1}}^{G}(u) \in \mathbf{Z}$. This follows from the fact that $p_{\Lambda, \Lambda^{\prime}}(q) \in \mathbf{Z}[q]$, and the description of $Y_{\Lambda}$ in Theorem 5.7. We have the following result.

Theorem 5.14. Assume that $G$ is a symplectic group. Let $r$ be a prime such that $r$ does not divide the order of $G^{F^{r}}$. For any $u \in G_{\text {uni }}^{F}$, we have

$$
Q_{L, C_{1}, \mathscr{E}_{1}, \varphi_{1}}^{G}(u) \equiv Q_{L, C_{1}, \mathscr{E}_{1}, \varphi_{1}^{r}}^{G}(u) \bmod r
$$


Proof. Take $(C, \mathscr{E})$ and let $\Lambda$ be the corresponding symbol. Here $(C, \mathscr{E})$ is $F$-stable. We choose a split element $u \in C^{F}$, and define the function $Y_{\Lambda}$ on $G_{\text {uni }}^{F}$ given as in Theorem 5.7. We can also define the corresponding function on $G_{\text {uni }}^{F^{r}}$ by using the same $u$, which we denote by $Y_{\Lambda}^{(r)}$. We note that

(5.14.1) Assume that $r$ is odd. Then for any $u \in C^{F}$, we have $Y_{\Lambda}(u)=Y_{\Lambda}^{(r)}(u)$.

Here $F$ acts trivially on $A_{G}(u)$, and for each $a \in A_{G}(u)$, let $u_{a}$ be a representative of the $G^{F}$-class in $C^{F}$ corresponding to $a \in A_{G}(u)$. Then $u_{a}$ is obtained as $u_{a}=g u g^{-1}$ for some $g \in G$ such that $\alpha=g^{-1} F(g) \in Z_{G}(u)$ is a lift of $a \in A_{G}(u)=Z_{G}(u) / Z_{G}^{0}(u)$ on $Z_{G}(u)$. Here we have

$$
g^{-1} F^{r}(g)=\alpha F(\alpha) \cdots F^{r-1}(\alpha) \in Z_{G}(u)
$$

Since $F$ acts trivially on $A_{G}(u)$, the image of $g^{-1} F^{r}(g)$ coincides with $a^{r} \in A_{G}(u)$ under the $\operatorname{map} Z_{G}(u) \rightarrow A_{G}(u)$. As $A_{G}(u) \simeq(\mathbf{Z} / 2 \mathbf{Z})^{c}$ for some $c$, we see that $a^{r}=a$ in $A_{G}(u)$. (5.14.1) follows from this.

Let $X_{\Lambda}$ be the function on $G_{\text {uni }}^{F}$ as in 5.3, and we denote by $X_{\Lambda}^{(r)}$ the corresponding function on $G_{\text {uni }}^{F^{r}}$. By Theorem 5.9, we can write them as

$$
\begin{aligned}
X_{\Lambda} & =\sum_{\Lambda^{\prime}} p_{\Lambda, \Lambda^{\prime}}(q) Y_{\Lambda^{\prime}}, \\
X_{\Lambda}^{(r)} & =\sum_{\Lambda^{\prime}} p_{\Lambda, \Lambda^{\prime}}\left(q^{r}\right) Y_{\Lambda^{\prime}}^{(r)} .
\end{aligned}
$$

Since $p_{\Lambda, \Lambda^{\prime}}(t) \in \mathbf{Z}[t]$, we have $p_{\Lambda, \Lambda^{\prime}}(q) \equiv p_{\Lambda, \Lambda^{\prime}}\left(q^{r}\right) \bmod r$ by Fermat's little theorem. If $r$ satisfies the condition in the theorem, then $r$ is odd, and so (5.14.1) holds. Thus by (5.14.2), we see that $X_{\Lambda}(u) \equiv X_{\Lambda}^{(r)}(u) \bmod r$. The theorem now follows from (5.3.3).

Remark 5.15. Let $G$ be a connected reductive group, and $Q_{L, C_{1}, \mathscr{E}_{1}, \varphi_{1}}^{G}$ be the generalized Green function given in 5.1. For each $F$-stable pair $\Lambda=(C, \mathscr{E}) \in \mathscr{N}_{G}$, the function $X_{\Lambda}$ can be defined by a similar formula as in (5.3.2), and (5.3.3) holds under a suitable modification (here and below we use symbols $\Lambda, \Lambda^{\prime}$, etc. just to denote the elements in $\mathscr{N}_{G}$ ). Lusztig's theorem (Theorem 5.5) gives, under this general situation, an algorithm of computing $P=\left(p_{\Lambda, \Lambda^{\prime}}\right)$. We note that

(5.15.1) There exists a polynomial $p_{\Lambda, \Lambda^{\prime}}(t) \in \mathbf{Z}[t] \operatorname{such}$ that $p_{\Lambda, \Lambda^{\prime}}\left(q^{r}\right)$ coincides with $p_{\Lambda, \Lambda^{\prime}}$ for $G^{F^{r}}$, under the choice of $r$ satisfying a certain congruence condition.

In fact, $\Omega$ can be defined in general by a similar formula as in (5.5.1), and it is regarded as a matrix of rational functions on $t$ evaluated at $q^{r}$ for $r$ satisfying the congruence condition. Then the equation $P \Lambda^{t} P=\Omega$ determines $P$ uniquely in the level of rational functions, as far as this algorithm works well, namely if the division by 0 does not occur in each step. But this is guaranteed by Theorem 5.5 which asserts that the evaluation at $t=q^{r}$ gives an algorithm of computing $P$. Thus $p_{\Lambda, \Lambda^{\prime}}(t) \in \mathbf{Q}(t)$. But we know by [L4, (24.5.2)] that $p_{\Lambda, \Lambda^{\prime}} \in \mathbf{Z}$. Hence (5.15.1) holds.

By using this result instead of Theorem 5.9, we can extend Theorem 5.14 to the case of special orthogonal groups as follows. Let $G$ be a special orthogonal group. Then thanks 
SHOJI

to [S5, Cor. 4.4], a similar formula as in (5.14.1) holds for $G$ if $r$ is prime to the order of $G^{F^{r}}$. Thus combined with (5.15.1), we have

(5.15.2) Let $G$ be a special orthogonal group. Then the generalized Green function $Q_{L, C_{1}, \mathscr{E}_{1}, \varphi_{1}}^{G}$ satisfies a similar formula as in Theorem 5.14.

\section{REFERENCES}

[AH] P. Achar and A. Henderson; Orbit closures in the enhanced nilpotent cone, Adv. in Math. 219 (2008), 27-62, Corrigendum, ibid. 228 (2011), 2984-2988.

[BMM] M. Broué, G. Malle and J. Michel, Generic blocks of finite reductive groups, Asterisque 212 (1993), $7-92$.

[FI] M. Finkelberg and A. Ionov; Kostka-Shoji polynomials and Lusztig's convolution diagram, Bull. Inst. Math. Acad. Sin. (NS) 13 (2018) no.1, 31 - 42.

[G] M. Geck; Green functions and a theorem of Hartley-Turull; preprint.

[K] S. Kato; An exotic Deligne-Langlands correspondence, Duke Math. J. 148 (2009), 306 - 371.

[L1] G. Lusztig; Irreducible representations of finite classical groups, Invent. Math. 43 (1977), 125 175.

[L2] G. Lusztig; Intersection cohomology complexes on a reductive group, Invent. Math.75 (1984), $205-272$.

[L3] G. Lusztig; Character sheaves, II, Adv. in Math. 57 (1985), 226 - 265.

[L4] G. Lusztig; Character sheaves, V, Adv. in Math. 61 (1986), 103 - 155.

[L5] G. Lusztig; On the character values of finite Chevalley groups at unipotent elements, J. Algebra 194 (1986), 146 - 194.

[LS] G. Lusztig and N. Spaltenstein; On the generalized Springer correspondence for classical groups, in "Algebraic Groups and Related Topics", Advanced Studies in Pure Math. 6, North-Holland and Kinokuniya, 1985, pp. $289-316$.

[Mac] I.G. Macdonald; "Symmetric functions and Hall polynomials", Claredon Press, Oxford, 1995.

[Mal] G. Malle; Unipotent Grade imprimitiver komplexer Spiegelungsgruppen, J. Algebra 177 (1995), $768-825$.

[S1] T. Shoji; On the Green polynomials of classical groups, Invent. Math., 74 (1983), 237 - 267.

[S2] T. Shoji; Green functions associated to complex reflection groups, J. Algebra 245 (2001), 650 694.

[S3] T. Shoji; Green functions associated to complex reflection groups, II, J. Algebra 258 (2002), 563 - 598 .

[S4] T. Shoji; Green functions attached to limit symbols, in "Representation theory of algebraic groups and quantum groups", Advanced Studies in Pure Math. 40, North-Holland and Kinokuniya, 2004, pp.443 - 467.

[S5] T. Shoji; Generalized Green functions and unipotent classes for finite reductive groups, II, Nagoya Math. J. 188 (2007), 133 - 170.

[S6] T. Shoji; Kostka functions associated to complex reflection groups and a conjecture of FinkelbergIonov, Science China Math. 61 (2018), 353 - 384.

[SS] T. Shoji and K. Sorlin; Exotic symmetric space over a finite field, I, Transformation Groups, 18 (2013), 877-929.

\section{T. Shoji}

School of Mathematical Sciences, Tongji University

1239 Siping Road, Shanghai 200092, P. R. China

E-mail: shoji@tongji.edu.cn 